TITLE:

\title{
A fast computational method for potential flows in multiply connected coastal domains
}

$\operatorname{AUTHOR}(\mathrm{S})$ :

Nasser, Mohamed M. S.; Sakajo, Takashi; Murid, Ali H. M.; Wei, Lee Khiy

\section{CITATION:}

Nasser, Mohamed M. S. ... [et al]. A fast computational method for potential flows in multiply connected coastal domains. Japan Journal of Industrial and Applied Mathematics 2015, 32(1):205-236

\section{ISSUE DATE:}

2015-02-06

URL:

http://hdl.handle.net/2433/198599

\section{RIGHT:}

The final publication is available at Springer via http://dx.doi.org/10.1007/s13160-0150168-6.; 許諾条件により本文ファイルは2016-02-06に公開.; This is not the published version. Please cite only the published version.; この論文は出版社版でありません。引用 の際には出版社版をご確認ご利用ください。 


\title{
A fast computational method for potential flows in multiply connected coastal domains
}

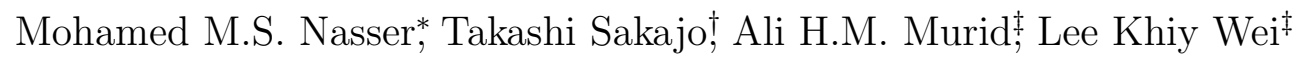

\begin{abstract}
We present a fast and accurate numerical method for constructing incompressible, inviscid and irrotational flows in two-dimensional coastal domains, which are unbounded multiply connected domains above an infinitely long coastline boundary. In the numerical method, we utilize a numerical conformal mapping method based on a boundary integral equation with the generalized Neumann kernel in order to construct conformal mappings from coastal domains onto five of Koebe's canonical domains. The numerical method is fast and accurate, since it just requires $O((m+1) n \ln n)$ operations and it converges with $O\left(e^{-c n}\right)$ for coastal domains of connectivity $m+1$, where $n$ is the number of nodes in discretizing each smooth boundary component and $c$ is a positive constant. With some examples, we also show that it is applicable to arbitrary coastal domains with high connectivity and complex geometry.
\end{abstract}

Keywords. Potential flows; Multiply connected domains; Generalized Neumann kernel; Numerical conformal mappings; Kirchhoff-Routh path function

MSC. 30C30, 76B07, 86A05, 76B47.

\section{Introduction}

The sea areas surrounding Penang Island and Japanese islands as shown in Figure 1 are represented by flow domains whose boundaries consist of a long coastline of a continent and offshore islands. In these mesoscale flow domains, we are concerned with how the existence of topographic obstacles affects the motion of a large eddy, which is a flow domain with a circulation, since the eddy transports mass, momentum and substances such as salt and minerals in oceans. For instance, Richardson and Tychensky observed a collision of a subsurface eddy to seamounts in the Canary Basin [26]. Fratantoni et al. [9] investigated whether a big ocean current, called North Brazil current ring, can pass the multiple gaps of southeastern Caribbean islands into the Gulf of Mexico. Moreover, it is important to investigate the interaction with not only topographic obstacles, but also a global sea current, an inflow from a river mouth and a flow induced by the other large eddies, since the eddy is also advected by these ambient flows.

In some flow domains along coastlines, the vertical flow scale is often sufficiently small compared to the horizontal one, and we thus regard the flow domains as unbounded multiply connected domains in the two-dimensional space exterior to an infinitely long coastline boundary and multiple offshore obstacles, which we refer to as coastal domains in the present paper. For the sake of theoretical convenience, we consider potential flows

\footnotetext{
* Department of Mathematics, Faculty of Science, King Khalid University, P. O. Box 9004, Abha, Saudi Arabia. E-mail: mms_nasser@hotmail.com

${ }^{\dagger}$ Department of mathematics, Kyoto University, Kyoto 606-8502, Japan. E-mail: sakajo@math.kyotou.ac.jp \& JST CREST, Japan Science and Technology Agency, Tokyo

${ }^{\ddagger}$ Department of Mathematical Sciences, Faculty of Science, Universiti Teknologi Malaysia, 81310 UTM Johor Bahru, Johor, Malaysia. E-mail: alihassan@utm.my, leekhiywei@gmail.com
} 
in coastal domains, in which the incompressible and inviscid flows are assumed to be irrotational except a finite number of point singularities. Then, by identifying the twodimensional space with the complex plane with $z=x+i y$, the velocity field of potential flows $(u(x, y), v(x, y))$ is obtained from an analytic function $W(z)$, called a complex potential, via $u-i v=W^{\prime}(z)$. In the theory of potential flows, we assume that a flow domain with a big circulation is confined into a point and that the vortex eddy is modelled as a point vortex whose velocity field is induced by a complex potential with a logarithmic singularity. Johnson and McDonald investigated the motion of a point vortex in a coastal domain whose boundaries consist of a straight coastline and a circular obstacle [11] and in a flow domain near multiple gaps between slit segments [12]. In these studies, since the boundary shapes of obstacles are simple, we can make the best use of analytic formulas of complex potentials and we are thus able to investigate the interactions between the vortex motion and the configurations of the obstacles qualitatively by analytic means.

(a)

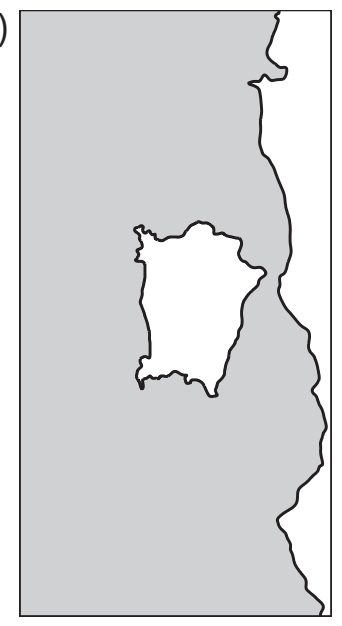

(b)

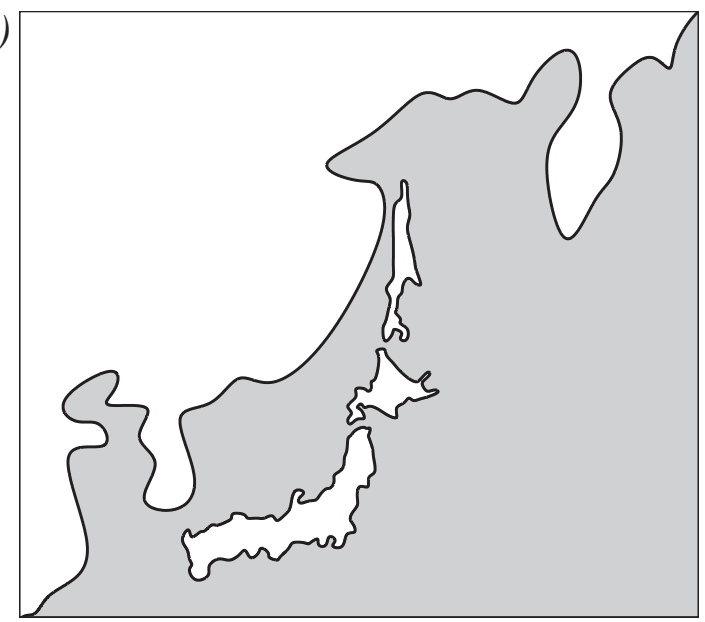

Figure 1: Coastal domains around (a) Penang Island and the coastline of west Malaysia, and (b) Japanese islands and the coastline of East Asia.

One extension of the preceding studies of potential flows is dealing with real topography of coastal domains as shown in Figure 1. It is in principle possible to derive complex potentials by constructing a conformal mapping from a given real coastal domain to a canonical multiply connected domain for which analytic expressions of complex potentials have already been known. However, in practice, it is extremely difficult to construct such a conformal mapping analytically when the shapes of boundaries become complicated. Hence we need to rely on numerical conformal mapping techniques. Numerical conformal mapping methods have been applied to many flow problems. For example, the particle charge simulation method developed by Amano et al. [1] was used to obtain complex potentials in multiply connected channel domains with boundaries of arbitrary shapes [29].

In the construction of potential flows, the numerical conformal mapping methods are required to be fast and accurate in order to consider coastal domains with many obstacles and with boundaries of arbitrary shapes. Examples of such numerical conformal mapping methods are presented in [19-23,25,32]. In [19-23], a unified method has been presented for computing the conformal mapping from multiply connected domains onto Koebe's 39 canonical slit domains as well as the canonical domain obtained by removing rectilinear slits from a strip. The method is based on a boundary integral equation with the generalized Neumann kernel. Discretizing the integral equation by the Nyström method with the trapezoidal rule yields an $(m+1) n \times(m+1) n$ linear system where $m+1$ is the multiplicity of the domain and $n$ is the number of nodes in the discretization of each boundary component of the domain. The order of the convergence of the Nyström method is based on the order of the convergence of the trapezoidal rule which in turn depends on the smoothness 
of the integrand [2, p. 109]. If the integrand is $q$ times continuously differentiable, then the rate of convergence of the trapezoidal rule is $O\left(1 / n^{q}\right)$. For infinitely continuously differentiable integrands, the rate of convergence is $O\left(e^{-c n}\right)$ where $c$ is a positive constant (see [13, p. 223]). The smoothness of the integrand depends on the smoothness of the boundary of the domain. For $C^{\infty}$ smooth boundaries, the integrands are infinitely continuously differentiable so very accurate results can be obtained. The $(m+1) n \times(m+1) n$ linear system can be solved by the generalized minimal residual (GMRES) method combined with the Fast Multipole Method (FMM) in $O((m+1) n \ln n)$ operations [23,24]. The method presented in $[25,32]$ is based on a boundary integral equations with the adjoint generalized Neumann kernel. A fast method for solving the integral equations with the adjoint generalized Neumann kernel is presented in [24].

The purpose of the present paper is constructing potential flows in multiply connected coastal domains fast and accurately by using these numerical conformal mapping methods. Let us first state the problem and then give a quick guide showing how to compute potential flows in Section 2. After constructing three preprocessing conformal mappings in Section 3 and four canonical conformal mappings in Section 4, we make use of these conformal mappings to compute potential flows in multiply connected coastal domains in Section 5. We also show some applications to the coastline of west Malaysia and the flow domain in the vicinity of East Asia in Figure 1. We then discuss the motion of a single point vortex as was done by Johnson and McDonald [11,12]. Final section is conclusion. In Appendices A and B, we review how to construct conformal mappings using the generalized Neumann kernel developed in $[20,22,25]$. Appendix C gives a technical note for the practical numerical computations.

\section{Problem setting and a quick guide to the solution}

Let us describe the problem considered in this paper and give a list of conformal mappings required to construct potential flows for the readers' reference. Figure 2 is a schematic picture of a multiply connected coastal domain in the complex $z$-plane, which is an unbounded domain above a wall (or a coastline) boundary extending to infinity containing $m$ obstacles. We focus, in particular, on the domain in the neighborhood of the part of the wall boundary, say $E_{0}$, between two points $z_{1}$ and $z_{2}$ on it. We assume that the boundaries of the obstacles $E_{1}, E_{2}, \ldots, E_{m}$ are smooth Jordan curves. Let $\alpha_{z}, \sigma_{z}$ denote fixed points in the coastal domain and $\beta_{z}$ be a fixed point on the wall boundary $E_{0}$, which can be chosen freely when we construct the conformal mappings.

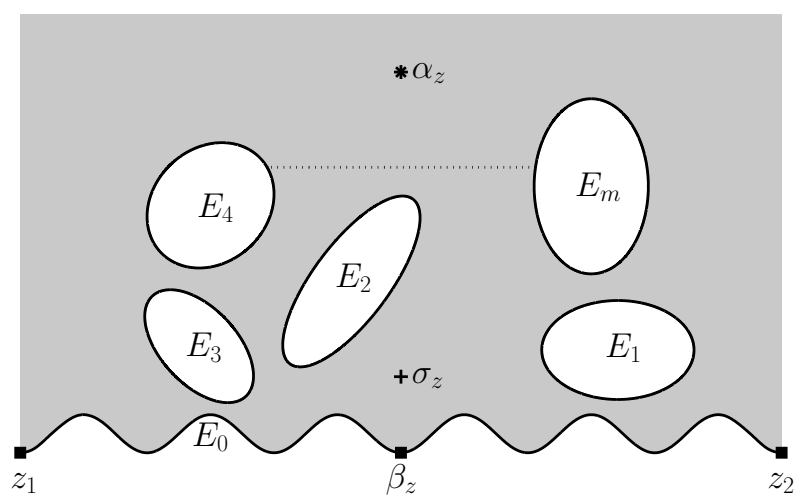

Figure 2: A coastal domain: a domain exterior to a curved wall (or a coastline) boundary $E_{0}$ and $m$ obstacles $E_{j}$ for $j=1, \ldots, m$.

We introduce three preprocessing conformal mappings $\zeta=H(z), \xi=\Phi(\zeta)$ and $\eta=$ $\Upsilon(\xi)$ in Section 3, and four canonical conformal mappings $w=\omega_{D}(\xi), w=\omega_{S}(\xi), w=$ 


\begin{tabular}{|c|c|c|}
\hline Potential flows & Conformal mappings & Subsections \\
\hline \hline Point vortex & $w=\omega_{D}(\xi), \xi=\Phi(\zeta), \zeta=H(z)$ & $3.1,3.2,4.1,5.1$ \\
\hline Source-sink pair & $w=\omega_{S}(\xi), \xi=\Phi(\zeta), \zeta=H(z)$ & $3.1,3.2,4.2,5.1$ \\
\hline Uniform flow & $w=\omega_{U}(\eta), \eta=\Upsilon(\xi), \xi=\Phi(\zeta), \zeta=H(z)$ & $3.1,3.2,3.3,4.3,5.1$ \\
\hline Source at boundary & $w=\omega_{B}(\eta), \eta=\Upsilon(\xi), \xi=\Phi(\zeta), \zeta=H(z)$ & $3.1,3.2,3.3,4.4,5.1$ \\
\hline Kirchhoff-Routh function & $w=\omega_{D}(\xi), \xi=\Phi(\zeta), \zeta=H(z)$ & $3.1,3.2,4.1,5.2$ \\
\hline
\end{tabular}

Table 1: List of conformal mappings used in order to construct complex potentials for various flow elements in multiply connected coastal domains and the subsections to be referred in this paper.

$\omega_{U}(\eta)$ and $w=\omega_{B}(\eta)$ in Section 4. Potential flows in this coastal domain are defined as their compositions. Table 1 shows which conformal mappings are required in order to construct the complex potentials for various flow elements. The conformal mappings $H(z)$ and $\Phi(\zeta)$ are expressed analytically, while the conformal mappings $\Upsilon(\xi), \omega_{D}(\xi)$, $\omega_{S}(\xi), \omega_{U}(\eta)$ and $\omega_{B}(\eta)$ are constructed by numerical means. On the construction of the conformal mappings, there arise Riemann-Hilbert problems and their solutions are obtained by solving a boundary integral equation with the generalized Neumann kernel explained in Appendices A and B.

\section{Preprocessing conformal mappings}

\subsection{Conformal mapping to the domain $\Lambda$}

Let $H$ be the linear transformation

$$
\zeta=H(z)=\frac{2}{z_{2}-z_{1}}\left(z-z_{1}\right)-1,
$$

which maps the coastal domain in the $z$-plane to a multiply connected domain $\Lambda$ in the $\zeta$-plane as shown in Figure 3(a). The wall boundary $E_{0}$ is transformed into a simple arc $L_{0}^{\prime}$ between -1 and +1 and the boundaries of the obstacles $E_{1}, E_{2}, \ldots, E_{m}$ are mapped to smooth Jordan curves $L_{1}, \ldots, L_{m}$. Let $L_{0}^{\prime \prime}$ be the part of the real line from $-\infty$ to -1 and $L_{0}^{\prime \prime \prime}$ be the part of the real line from +1 to $+\infty$. We assume that the part of the wall on the left of $z_{1}$ is mapped to $L_{0}^{\prime \prime}$ and the part of the wall on the right of $z_{2}$ is mapped to $L_{0}^{\prime \prime \prime}$. The images of $\alpha_{z}$ and $\sigma_{z}$ are $\alpha_{\zeta}$ and $\sigma_{\zeta} \in \Lambda$, respectively, and $H\left(\beta_{z}\right)=\beta_{\zeta} \in L_{0}^{\prime}$.

Let $L_{0}$ be an infinite simple curve in the $\zeta$-plane that consists of three parts $L_{0}^{\prime}, L_{0}^{\prime \prime}$ and $L_{0}^{\prime \prime \prime}$, i.e., $L_{0}=L_{0}^{\prime} \cup L_{0}^{\prime \prime} \cup L_{0}^{\prime \prime \prime}$. Then $\Lambda$ becomes a multiply connected domain obtained by removing $m$ simply connected domains from the half $\zeta$-plane above $L_{0}$. The boundary $L$ of the domain $\Lambda$ is given by $L=L_{0} \cup L_{1} \cup \cdots \cup L_{m}$. The orientation of the boundary $L$ is taken so that the domain $\Lambda$ is always on the left-hand side of $L$, which are shown in Figure 3(a) by bold arrows.

\subsection{Conformal mapping to the domain $D$}

In the second step, we map the domain $\Lambda$ to a bounded multiply connected domain $D$ in Figure 3(b). We introduce the Möbius transform

$$
\xi=\Phi(\zeta)=\frac{1+\mathrm{i} \tau \zeta}{\zeta+\mathrm{i} \tau},
$$

where a positive real constant $\tau$ is chosen so that $-\mathrm{i} \tau$ is located below $L_{0}$ and $\mathrm{i} / \tau$ located above $L_{0}$. The Möbius transform $\xi=\Phi(\zeta)$ maps $\Lambda$ in the $\zeta$-plane to the bounded domain 

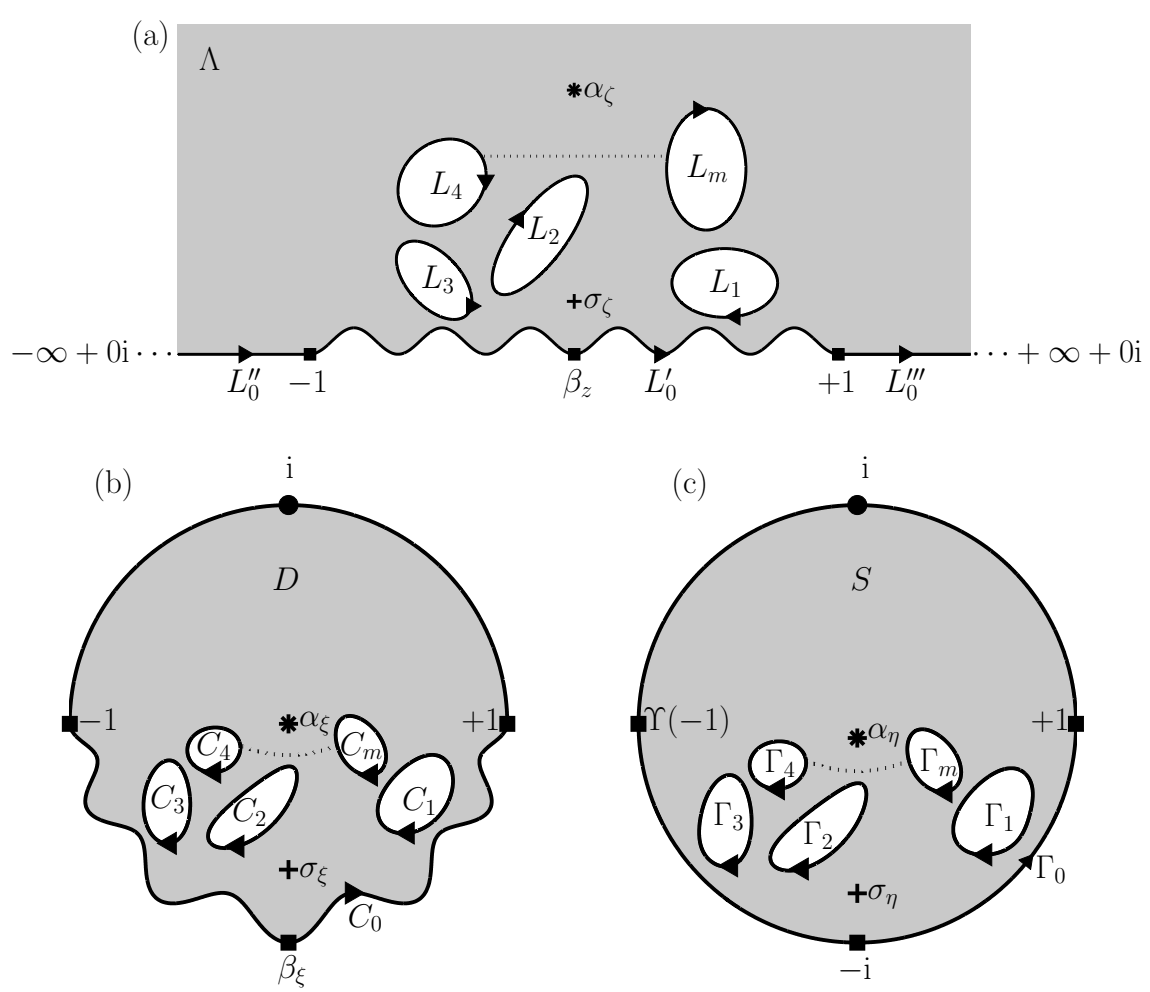

Figure 3: (a) An unbounded multiply connected domain $\Lambda$ in the $\zeta$-plane. (b) A bounded multiply connected domain $D$ in the $\xi$-plane. (c) A bounded domain inside the unit circle $S$ in the $\eta$-plane.

$D$ in the $\xi$-plane and the image of the boundary $L_{0}$ of $\Lambda$ becomes a closed curve, say $C_{0}$. For $j=1, \ldots, m, C_{j}=\Phi\left(L_{j}\right)$ are closed smooth Jordan curves inside $D$. Thus the boundary $C$ of the domain $D$ becomes $C=C_{0} \cup C_{1} \cup \cdots \cup C_{m}$. The orientation of the boundary is such that the domain $D$ is on the left-hand side of $C$.

In this paper, we may assume that $\tau=1$, without loss of generality, i.e., i is always above $L_{0}$ and $-\mathrm{i}$ is always below $L_{0}$. Hence, the function $\Phi$ satisfies $\Phi(\infty)=\mathrm{i}, \Phi(-1)=-1$ and $\Phi(1)=1$. The mapping function $\xi=\Phi(\zeta)$ maps the points $\alpha_{\zeta}, \sigma_{\zeta}$ in $\Lambda$ to points $\alpha_{\xi}=\Phi\left(\alpha_{\zeta}\right), \sigma_{\xi}=\Phi\left(\sigma_{\zeta}\right)$ in $D$ and the point $\beta_{\zeta}$ on $L_{0}$ to the point $\beta_{\xi}$ on $C_{0}$, respectively. See Figure 3(b).

\subsection{Conformal mapping to the domain $S$}

In order to compute the conformal mappings to the canonical domains $\Omega_{U}$ and $\Omega_{B}$ in Section 4 , we need another mapping from the domain $D$ to a bounded multiply connected domain $S$ whose external boundary is the unit circle. This can be done by mapping the simply connected domain inside $C_{0}$ onto the unit disc. According to [20], the Riemann mapping function $R(\zeta)$ that maps the boundary of the simply connected domain inside $C_{0}$ onto the unit disk is represented by

$$
R(\xi)=c\left(\xi-\alpha_{\xi}\right) e^{\left(\xi-\alpha_{\xi}\right) \hat{f}(\xi)},
$$

where $c$ is a real positive constant. Since the boundary $C_{0}$ is mapped to the unit circle, the boundary condition of this function is given by $|R(\xi)|=1$ on $\xi \in C_{0}$. Taking the logarithm of the absolute value of (3) and evaluating it on the boundary $C_{0}$, we obtain the equation for the function $f$ on the boundary $C_{0}$,

$$
\operatorname{Re}\left[\left(\xi-\alpha_{\xi}\right) \stackrel{\circ}{f}(\xi)\right]=\stackrel{\gamma}{\gamma}(\xi)+\stackrel{\circ}{h}(\xi),
$$


where

$$
\stackrel{\gamma}{\gamma}(\xi)=-\log \left|\xi-\alpha_{\xi}\right|, \quad \stackrel{\circ}{h}(\xi)=\log \frac{|R(\xi)|}{c}=\log \frac{1}{c} .
$$

This is a Riemann-Hilbert problem, whose unique solution is given by solving the boundary integral equation with the generalized Neumann kernel explained in Appendix B. In addition, let $T(z)$ be the Möbius transformation that maps the unit disk onto itself and the points $R(1), R(\mathrm{i})$ and $R\left(\beta_{\xi}\right)$ into 1 , i and $-\mathrm{i}$, respectively, i.e.,

$$
T(z)=\frac{(1-\mathrm{i})\left(z-R\left(\beta_{\xi}\right)\right)(R(i)-R(1))+\mathrm{i}(z-R(1))\left(R(\mathrm{i})-R\left(\beta_{\xi}\right)\right)}{(1-\mathrm{i})\left(z-R\left(\beta_{\xi}\right)\right)(R(i)-R(1))-(z-R(1))\left(R(\mathrm{i})-R\left(\beta_{\xi}\right)\right)} .
$$

Then the function

$$
\Upsilon(\xi)=T(R(\xi))
$$

conformally maps the simply connected domain inside $C_{0}$ to the unit disk with $\Upsilon(1)=1$, $\Upsilon(\mathrm{i})=\mathrm{i}$ and $\Upsilon\left(\beta_{\xi}\right)=-\mathrm{i}$. Hence, the conformal mapping $\eta=\Upsilon(\xi)$ maps the domain $D$ in the $\xi$-plane onto a multiply connected domain $S$ in the $\eta$-plane with the boundary $\Gamma=\Gamma_{0} \cup \Gamma_{1} \cup \cdots \cup \Gamma_{m}$ in Figure $3(\mathrm{c})$. The boundary $\Gamma_{0}$ is the unit circle and the other boundaries $\Gamma_{j}=\Upsilon\left(C_{j}\right)$ for $j=0,1, \ldots, m$ are smooth Jordan curves inside $\Gamma_{0}$. The orientation of the boundary $\Gamma$ is chosen so that $S$ is on the left-hand side of $\Gamma$. The function $\Upsilon$ maps the point $\alpha_{\xi}$ in $D$ to a point $\alpha_{\eta}$ in $S$ and maps the three points 1 , i and $\beta_{\xi}$ on $C_{0}$ to the three points $1, \mathrm{i}$ and $-\mathrm{i}$ on $\Gamma_{0}$, respectively.

\section{Conformal mappings to canonical domains}

\subsection{Conformal mapping to the unit disk with circular slits $\Omega_{D}$}

Let $\Omega_{D}$ be a canonical multiply connected domain inside the unit circle with $m$ finite circular slits $B_{j}$ for $j=1, \ldots, m$ as shown in Figure 4(a). We construct the conformal mapping $w=\omega_{D}(\xi)$ from the domain $D$ in the $\xi$-plane onto a canonical domain $\Omega_{D}$ in the $w$-plane satisfying the following boundary condition:

$$
\left|\omega_{D}(\xi)\right|=R_{j}, \quad \xi \in C_{j}, j=0,1, \ldots, m,
$$

where $R_{0}=1$ and $R_{1}, R_{2}, \ldots, R_{m}$ are unknown real constants. According to [20, 4.2] , the mapping function $\omega_{D}$ can be written as

$$
w=\omega_{D}(\xi)=c\left(\xi-\alpha_{\xi}\right) e^{\left(\xi-\alpha_{\xi}\right) f(\xi)},
$$

where $c$ is a real constant and $f$ is an auxiliary analytic function on $D$. By taking the logarithm of the absolute value of (7) and evaluating it on the boundary $C=C_{0} \cup C_{1} \cup$ $\cdots \cup C_{m}$ of the domain $D$ with (6), we obtain the following Riemann-Hilbert problem.

$$
\operatorname{Re}\left[\left(\xi-\alpha_{\xi}\right) f(\xi)\right]=\gamma(\xi)+h(\xi), \quad \xi \in C,
$$

where

$$
\gamma(\xi)=-\log \left|\xi-\alpha_{\xi}\right|, \quad h(\xi)=\log \frac{\left|\omega_{D}(\xi)\right|}{c}=\left(\log \frac{1}{c}, \log \frac{R_{1}}{c}, \ldots, \log \frac{R_{m}}{c}\right) .
$$

The unique solution of this problem is obtained by solving the boundary integral equation with the generalized Neumann kernel in Appendix A. It gives us the boundary value of $f$ and the constants $c, R_{j}$ for $j=1, \ldots, m$. The value of the function $f(\xi)$ in the interior points of the domain $D$ is evaluated from its boundary value through Cauchy's integral formula. 

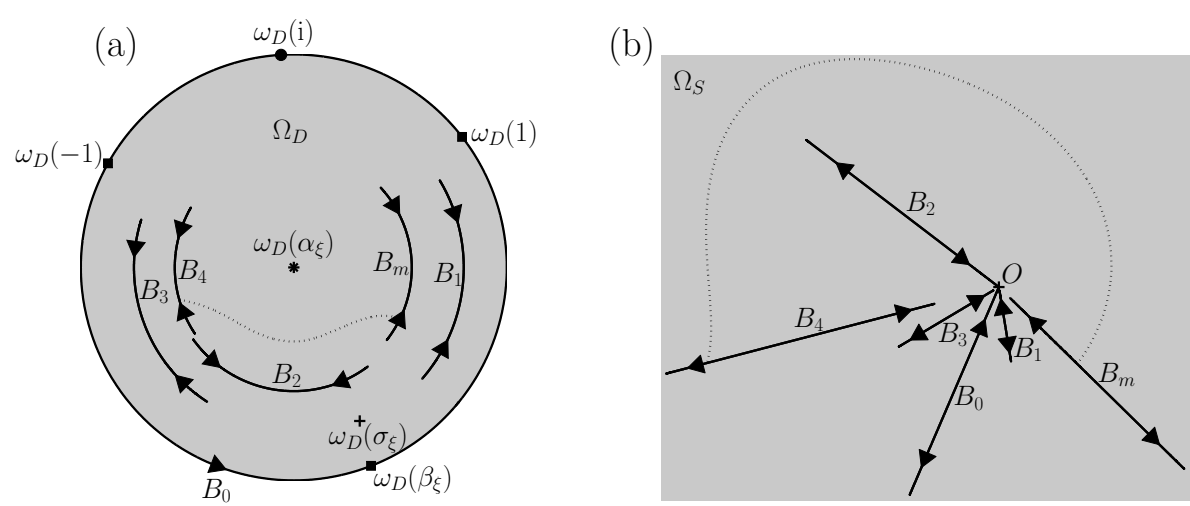

Figure 4: Canonical multiply connected domains in the $w$-plane; (a) $\Omega_{D}$ and (b) $\Omega_{S}$.

\subsection{Conformal mapping to radial slits domain $\Omega_{S}$}

Figure 4(b) shows a canonical domain $\Omega_{S}$ in the $w$-plane with $m+1$ finite radial slits $B_{j}$ for $j=0,1, \ldots, m$ emanating from the origin. Let $w=\omega_{S}(\xi)$ be the conformal mapping from the domain $D$ in the $\xi$-plane onto $\Omega_{S}$. The boundary condition of this function $\omega_{S}$ is given by

$$
\arg \left(\omega_{S}\left(\xi_{j}\right)\right)=R_{j}, \quad \xi \in C_{j}, j=0,1, \ldots, m,
$$

where $R_{0}, R_{1}, \ldots, R_{m}$ are some real constants. It is shown in $[20, \S 4.4]$ that the function $\omega_{S}$ is written as

$$
\omega_{S}(\xi)=\left(\frac{1}{\xi-\alpha_{\xi}}-\frac{1}{\sigma_{\xi}-\alpha_{\xi}}\right) e^{\mathrm{i}\left(\xi-\alpha_{\xi}\right) f(\xi)},
$$

where $f$ is an auxiliary analytic function on $D$. Taking the argument of both sides of (10) and evaluating it on the boundary $C=C_{0} \cup C_{1} \cup \cdots \cup C_{m}$ of the domain $D$ with (9) give rise to the following Riemann-Hilbert problem.

$$
\operatorname{Re}\left[\left(\xi-\alpha_{\xi}\right) f(\xi)\right]=\gamma(\xi)+h(\xi), \quad \xi \in C,
$$

where

$$
\gamma(\xi)=-\arg \left(\frac{1}{\xi-\alpha_{\xi}}-\frac{1}{\sigma_{\xi}-\alpha_{\xi}}\right), \quad h(\xi)=\arg \left(\omega_{S}(\xi)\right)=\left(R_{0}, R_{1}, \ldots, R_{m}\right) .
$$

The unique solution of this problem is again obtained by solving the boundary integral equation with the generalized Neumann kernel in Appendix A, which gives the boundary value of $f(\xi)$ and the constants $R_{j}$ for $j=0,1, \ldots, m$.

\subsection{Conformal mapping to the half plane with horizontal slits $\Omega_{U}$}

Figure 5 shows a canonical domain $\Omega_{U}$ in the upper half $w$-plane $\operatorname{Im} w>0$ with $m$ horizontal rectilinear slits $B_{j}$ for $j=1, \ldots, m$. Let $w=\omega_{U}(\eta)$ be the conformal mapping from the bounded multiply connected domain $S$ in the $\eta$-plane onto the canonical domain $\Omega_{U}$ satisfying the following boundary condition on $\Gamma=\Gamma_{0} \cup \Gamma_{1} \cup \cdots \cup \Gamma_{m}$.

$$
\operatorname{Im}\left[\omega_{U}(\eta)\right]=R_{j}, \quad \eta \in \Gamma_{j}, j=0,1, \ldots, m,
$$

where $R_{0}=0$ and $R_{1}, R_{2}, \ldots, R_{m}$ are some real constants. According to [22, §4.5], the function $\omega_{U}$ can be written in the form of

$$
w=\omega_{U}(\eta)=\Psi(\eta)+\mathrm{i}\left(\eta-\alpha_{\eta}\right) f(\eta)-\mathrm{i} c,
$$


where $c$ is an unknown real constant, $f$ is an auxiliary analytic function in $S$, and $\Psi$ is given by

$$
\Psi(\eta)=\frac{1-\mathrm{i} \eta}{\eta-\mathrm{i}}
$$

Evaluating the imaginary part of (14) on the boundary $\Gamma=\Gamma_{0} \cup \Gamma_{1} \cup \cdots \cup \Gamma_{m}$ with (13) yields the following Riemann-Hilbert problem.

$$
\operatorname{Re}\left[\left(\eta-\alpha_{\eta}\right) f(\eta)\right]=\gamma(\eta)+h(\eta), \quad \eta \in \Gamma,
$$

where

$$
\gamma(\eta)=-\operatorname{Im}[\Psi(\eta)], \quad h(\eta)=\operatorname{Im}\left[\omega_{U}(\eta)\right]+c=\left(c, R_{1}+c, \ldots, R_{m}+c\right) .
$$

It is clear from (15) that $\Psi(\eta)$ is on the real line when $\eta \in \Gamma_{0}$. Hence, $\gamma(\eta)=-\operatorname{Im}[\Psi(\eta)]=$ 0 for $\eta \in \Gamma_{0}$, i.e., the function $\gamma$ is given by

$$
\gamma(\eta)= \begin{cases}0, & \eta \in \Gamma_{0}, \\ -\operatorname{Im}[\Psi(\eta)], & \eta \in \Gamma_{j}, j=1, \ldots, m .\end{cases}
$$

The boundary integral equation with the generalized Neumann kernel in Appendix A with $\xi$ being replaced by $\eta$ gives the solution of this problem. We thus obtain the boundary value of $f$ and the constants $c, R_{j}$ for $j=1, \ldots, m$. The value of the function $f(\eta)$ in the interior points of the domain $S$ can be evaluated from its boundary values through Cauchy's integral formula.

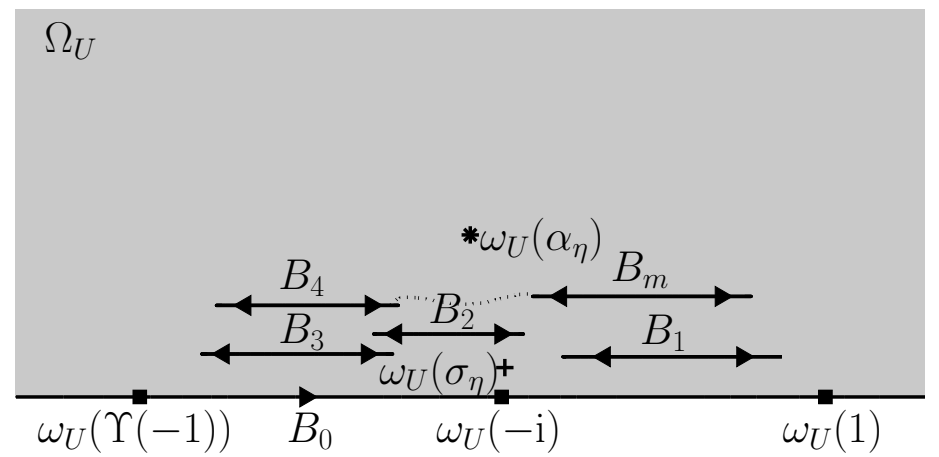

Figure 5: The canonical multiply connected domain $\Omega_{U}$ in the $w$-plane.

\subsection{Conformal mapping to the half plane with radial slits $\Omega_{B}$}

An upper half plane $\operatorname{Im} w>0$ in the $w$-plane containing $m$ finite radial slits $B_{j}$ for $j=1, \ldots, m$ is denoted by $\Omega_{B}$ as shown in Figure 6 . We construct the conformal mapping $w=\omega_{B}(\eta)$ from the domain $S$ in the $\eta$-plane onto the canonical domain $\Omega_{B}$ with the following boundary conditions:

$$
\begin{array}{ll}
\operatorname{Im}\left[\omega_{B}(\eta)\right]=0, & \eta \in \Gamma_{0}, \\
\arg \left[\omega_{B}(\eta)\right]=R_{j}, \quad \eta \in \Gamma_{j}, j=1, \ldots, m,
\end{array}
$$

where $R_{1}, R_{2}, \ldots, R_{m}$ are some real constants. The function $\omega_{B}$ can be expressed as $[22$, $\S 4.6]$

$$
w=\omega_{B}(\eta)=\Psi(\eta) e^{\mathrm{i}\left(\eta-\alpha_{\eta}\right) f(\eta)-\mathrm{i} c},
$$

where $\Psi$ is given by (15), $c$ is a real constant, and $f$ is an auxiliary analytic function in $S$. Taking the logarithm of (18), we obtain

$$
\log \omega_{B}(\eta)=\log \Psi(\eta)+\mathrm{i}\left(\eta-\alpha_{\eta}\right) f(\eta)-\mathrm{i} c .
$$


Owing to (15), we have $\Psi(\eta)$ is real for $\eta \in \Gamma_{0}, \Psi(-\mathrm{i})=0$ and $\Psi(\mathrm{i})=\infty$. Similarly, it follows from the boundary condition (17) and the functional form (18) that $\omega_{B}(\eta)$ is real for $\eta \in \Gamma_{0}, \omega_{B}(-\mathrm{i})=0$, and $\omega_{B}(\mathrm{i})=\infty$. Since the orientation of $\Gamma_{0}$ is taken so that $S$ is on the left of $\Gamma_{0}$, then the orientation of both curves $\Psi\left(\Gamma_{0}\right)$ and $\omega_{B}\left(\Gamma_{0}\right)$ goes so that the upper half plane is on their left. Hence, for $\eta \in \Gamma_{0}$, the real functions $\Psi(\eta)$ and $\omega_{B}(\eta)$ have the same sign. Hence, by taking the imaginary part of (19) on the boundary $\Gamma_{0}$, we obtain

$$
\operatorname{Re}\left[\left(\eta-\alpha_{\eta}\right) f(\eta)\right]=c, \quad \eta \in \Gamma_{0} .
$$

For $\eta \in \Gamma_{j}, j=1,2, \ldots, m$, taking the imaginary part of both sides of (19) and using (17), we obtain

$$
\operatorname{Re}\left[\left(\eta-\alpha_{\eta}\right) f(\eta)\right]=-\operatorname{Im}[\log \Psi(\eta)]+R_{j}+c, \quad \eta \in \Gamma_{j} .
$$

Hence, the equations (20) and (21) become the following Riemann-Hilbert problem,

$$
\operatorname{Re}\left[\left(\eta-\alpha_{\eta}\right) f(\eta)\right]=\gamma(\eta)+h(\eta), \quad \eta \in \Gamma,
$$

where

$$
\gamma(\eta)=\left\{\begin{array}{ll}
0, & \eta \in \Gamma_{0}, \\
-\operatorname{Im}[\log \Psi(\eta)], & \eta \in \Gamma_{j}, j=1, \ldots, m,
\end{array}, \quad h(\eta)=\left(c, R_{1}+c, \ldots, R_{m}+c\right) .\right.
$$

The solution of this problem is obtained by solving the boundary integral equation with the generalized Neumann kernel in Appendix A with $\xi$ being replaced by $\eta$, which gives rise to the boundary value of $f$ and the constants $c, R_{j}$ for $j=1, \ldots, m$. The value of the function $f(\eta)$ in the interior points of the domain $S$ can be evaluated from its boundary values through Cauchy's integral formula.

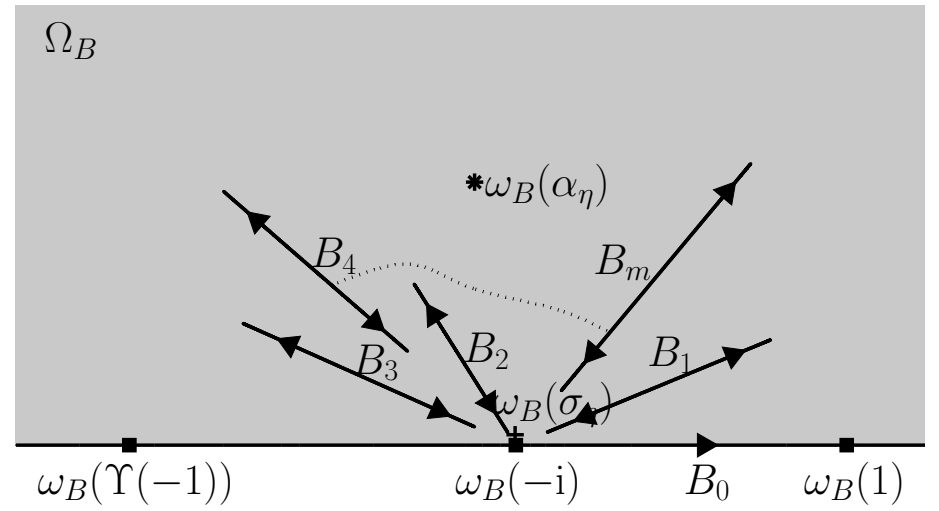

Figure 6: The canonical multiply connected domain $\Omega_{B}$ in the $w$-plane.

\section{Potential flows in the multiply connected coastal domains}

\subsection{Complex potentials}

According to the theory of potential flows, the level curves of $\operatorname{Im}[W(z)]$ for a given complex potential $W(z)$ represent streamlines of the potential flow. Hence, when we construct complex potentials using the conformal mappings in Sections 3 and 4, it is necessary to check whether they have the same singularity as the complex potentials in the unbounded domain without boundary and the imaginary part of $W(z)$ takes a constant value on each boundary of the domain so that the streamline coincides with the boundary.

Let us remember that $\frac{1}{2 \pi \mathrm{i}} \log \left(z-\alpha_{z}\right)$ represents the complex potential of a point vortex located at $z=\alpha_{z}$ in the unbounded complex $z$-plane without boundary. It follows from 
$\alpha_{\xi}=\Phi\left(H\left(\alpha_{z}\right)\right)$ and $(7)$ that the analytic function

$$
W_{D}(z)=\frac{1}{2 \pi \mathrm{i}} \log F_{D}\left(z ; \alpha_{z}\right), \quad F_{D}\left(z ; \alpha_{z}\right)=\omega_{D}(\Phi(H(z))),
$$

has the same logarithmic singularity in the neighborhood of $z=\alpha_{z}$ and its imaginary part becomes $\frac{1}{2 \pi} \log R_{j}$ on the boundary $E_{j}$ of the coastal domain for $j=0, \ldots, m$ owing to (6), which indicates that (23) represents the complex potential for a point vortex at $z=\alpha_{z}$. When there are $N$ point vortices at $\alpha_{z_{1}}, \alpha_{z_{2}}, \ldots, \alpha_{z_{N}}$ with strengths $\kappa_{1}, \kappa_{2}, \ldots$, $\kappa_{N}$, its complex potential is given by

$$
W(z)=\frac{1}{2 \pi \mathrm{i}} \sum_{j=1}^{N} \kappa_{j} \log F_{D}\left(z ; \alpha_{z_{j}}\right) .
$$

The analytic function

$$
W_{S}(z)=\frac{1}{2 \pi} \log \omega_{S}(\Phi(H(z)))
$$

has the same logarithmic singularities as the complex potentials $\frac{1}{2 \pi} \log \left(z-\sigma_{z}\right)$ for a $\operatorname{sink}$ and $-\frac{1}{2 \pi} \log \left(z-\alpha_{z}\right)$ for a sink in the neighborhood of $z=\sigma_{z}$ and $\alpha_{z}$, since we have $\alpha_{\xi}=\Phi\left(H\left(\alpha_{z}\right)\right), \sigma_{\xi}=\Phi\left(H\left(\sigma_{z}\right)\right)$ and (10). In addition, $\operatorname{Im}\left[W_{S}(z)\right]$ becomes $\frac{1}{2 \pi} \log R_{j}$ on the boundary $E_{j}$ for $j=0, \ldots, m$ owing to (9). Hence, the function represents the complex potential for the flow of a source-sink pair located at $z=\alpha_{z}$ and $\sigma_{z}$, respectively.

The complex potential for the uniform flow in the unbounded complex plane without boundary is represented by $U z$ for a complex constant $U$, which indicates that it has a pole singularity in the neighborhood of infinity. Owing to $\mathrm{i}=\Phi(H(\infty)),(14)$ and (15), the function

$$
W_{U}(z)=\omega_{U}(\Upsilon(\Phi(H(z))))
$$

has the pole singularity at infinity and the boundary value of $\operatorname{Im}\left[W_{U}(z)\right]$ becomes constant on each $E_{j}$. Hence, the function represents the complex potential for a uniform flow in the $z$-plane.

Finally, it follows from (1), (2) and (18) that we have $\Psi\left(\Upsilon\left(\Phi\left(H\left(\beta_{z}\right)\right)\right)=0\right.$. Then the function

$$
W_{B}(z)=\frac{1}{2 \pi} \log \omega_{B}(\Upsilon(\Phi(H(z))))
$$

gives rise to the complex potential for the flow induced by a source (or a sink) located at $z=\beta_{z}$ on the wall boundary of the coastal domain in the $z$-plane, since it has the same singularity as the source in the neighborhood of $z=\beta_{z}$ and the boundary value of its imaginary part becomes constant on each $E_{j}$ owing to (17).

As an example, we consider a coastal domain with five elliptic obstacles, in which we show streamlines for a uniform flow in Figure 7(a), a source point in Figure 7(b), a source-sink pair in Figure 7(c), and a vortex point in Figure 7(d), respectively. Figure 7(e) shows the streamline of the complex potential

$$
U W_{U}(z)+\frac{1}{2 \pi \mathrm{i}} \sum_{j=1}^{20} \kappa_{j} \log F_{D}\left(z ; \alpha_{z_{j}}\right)
$$

where $U=1.5$ and $\kappa_{j}= \pm 1$ with $\sum_{j=1}^{20} \kappa_{j}=0$.

\subsection{The Kirchhoff-Routh path function}

Lin $[15,16]$ considered a special Green function, the hydrodynamic Green's function $G\left(z ; z_{0}\right)$, for a domain $\mathcal{D}$ in the $z$-plane with a logarithmic singularity at $z=z_{0}$. The Hamiltonian $H\left(z_{1}, z_{2}, \ldots, z_{N}\right)$ describing the motion of $N$-point vortices located at $z_{m} \in \mathcal{D}$ with 
the strength $\kappa_{m} \in \mathbb{R}$ for $m=1, \ldots, N$, called the Kirchhoff-Routh path function [27], is constructed from the Green function as follows:

$$
H\left(z_{1}, z_{2}, \ldots, z_{N}\right)=\sum_{m=1}^{N} \sum_{k=m+1}^{N} \kappa_{m} \kappa_{k} G\left(z_{m} ; z_{k}\right)-\frac{1}{2} \sum_{m=1}^{N} \kappa_{m}^{2} g\left(z_{m} ; z_{m}\right) .
$$

Here, the last term $g\left(z_{m} ; z_{m}\right)$ is called the Robin function and it is defined by

$$
g\left(z_{m} ; z_{m}\right)=\lim _{z \rightarrow z_{m}} g\left(z ; z_{m}\right),
$$

where

$$
g\left(z ; z_{m}\right)=-G\left(z ; z_{m}\right)-\frac{1}{2 \pi} \log \left|z-z_{m}\right|
$$

is a harmonic function in the domain $\mathcal{D}$.

According to Crowdy and Marshall [5], the hydrodynamic Green function $G\left(z ; z_{0}\right)$ for unbounded domains with boundaries extending to infinity, which are referred to as coastal domains in this paper, satisfies the following boundary conditions.

$$
\begin{aligned}
G\left(z ; z_{0}\right) & =A_{j}, \quad \text { on } \quad E_{j}, \quad j=1, \ldots, m, \\
\oint_{E_{j}} \frac{\partial G}{\partial n} d s & =0, \quad j=1, \ldots, m, \\
G\left(z ; z_{0}\right) & =0, \quad \text { on } E_{0}, \\
G\left(z ; z_{0}\right) & =o(1), \quad \text { on a very large circle of radius } r_{0},
\end{aligned}
$$

for some real constants $A_{j}$ for $j=1, \ldots, m$. Let us recall that the imaginary part of the complex potential of a point vortex $W_{D}(z)$ is harmonic except the logarithmic singularity at $z=\alpha_{z}$ and it takes constant values along the boundaries of obstacles $E_{j}$ for $j=0, \ldots, m$ in coastal domains. Hence, the hydrodynamic Green function in coastal domains is given by

$$
G\left(z ; \alpha_{z}\right)=-\frac{1}{2 \pi} \log \left|F_{D}(z)\right| .
$$

Consequently, owing to $F_{D}\left(\alpha_{z}\right)=0$, the Robin function is represented by

$$
g\left(\alpha_{z} ; \alpha_{z}\right)=\lim _{z \rightarrow \alpha_{z}} g\left(z ; \alpha_{z}\right)=\lim _{z \rightarrow \alpha_{z}} \frac{1}{2 \pi} \log \left|\frac{F_{D}(z)-F_{D}\left(\alpha_{z}\right)}{z-\alpha_{z}}\right|=\frac{1}{2 \pi} \log \left|\frac{\mathrm{d} F_{D}}{\mathrm{~d} z}\left(\alpha_{z}\right)\right|,
$$

where the point $\alpha_{z}$ is taken arbitrarily in the flow domain. For each point $\alpha_{z}$, in order to compute the Robin function $g\left(\alpha_{z} ; \alpha_{z}\right)$, we need to compute the conformal mapping $w=F_{D}(z)$ which maps $\alpha_{z}$ in $z$-plane to the origin of $w$-plane. Thus, by the chain rule, we have

$$
g\left(\alpha_{z} ; \alpha_{z}\right)=\frac{1}{2 \pi} \log \left|\frac{\mathrm{d} \omega_{D}}{\mathrm{~d} \xi}\left(\alpha_{\xi}\right) \frac{\mathrm{d} \Phi}{\mathrm{d} \zeta}\left(\alpha_{\zeta}\right) \frac{\mathrm{d} H}{\mathrm{~d} z}\left(\alpha_{z}\right)\right|=\frac{1}{2 \pi} \log \left|c \frac{\mathrm{d} \Phi}{\mathrm{d} \zeta}\left(\alpha_{\zeta}\right) \frac{\mathrm{d} H}{\mathrm{~d} z}\left(\alpha_{z}\right)\right|,
$$

where $c$ is the real constant obtained by solving (8).

The Robin function is available to describing the motion of a single point vortex, since it has been shown in [5] that the trajectory of this vortex corresponds to a contour line of

$$
H\left(\alpha_{z}\right)=-\frac{\kappa^{2}}{2} g\left(\alpha_{z} ; \alpha_{z}\right),
$$

where $\kappa$ denotes the strength of the point vortex. We show in Figure 7 (f) contour plots of (35) for $\kappa=1$ in the coastal domain with five elliptic obstacles. Each contour line corresponds to an orbit of a single point vortex. 


\subsection{Accuracy and efficiency of the numerical methods}

\subsubsection{Comparison with analytic formula}

We compare the numerical results computed by the present method with those by the analytic formula. As a test bed, we consider the three coastal domains with a straight coastline that contain three circular obstacles in the complex $z$-plane as shown in Figures 8(a)-(c). Under the Möbius transformation

$$
\xi=\Phi(H(z))=\frac{\mathrm{i} z-5 \mathrm{i}}{z+5},
$$

these domains become the pre-images of the circular regions interior to the unit circle with circular inner boundaries in the complex $\xi$-plane, for which we have the analytic formula of complex potentials in terms of the Schottky-Klein prime function [5,6]. The SchottkyKlein prime function is computed by using the MATLAB code with an approximation parameter $N[7]$.

We use the formula in [5] to evaluate the complex potential $W_{D}(z)$ for the two coastal domains in Figures 8(a)-(b) for a point vortex at $\alpha_{z}=1.5+1.25$ i. Let $W_{D, n}\left(z_{k, j}\right)$ be the numerical approximation of $W_{D}\left(z_{k, j}\right)$ obtained by the present numerical method at $n$ discretization points along the boundary $L_{j}$, i.e. $z_{k, j} \in L_{j}$ for $k=1, \ldots, n$ and $j=0, \ldots, m$. Then, the maximum error is defined as follows.

$$
E_{\infty}^{(n, m)}=\max _{0 \leq j \leq m} \max _{1 \leq k \leq n}\left|\operatorname{Im}\left[W_{D, n}\left(z_{k, j}\right)\right]-\operatorname{Im}\left[W_{D}\left(z_{k, j}\right)\right]\right| .
$$

We then compute the Kirchhoff-Routh path function for the two coastal domains in Figures 8(a)-(b) with the analytic formula given in [6]. Since this function diverges along the boundaries, we pick 100 points in the coastline domain, which are shown as dots in Figure 8), and compute the maximum of differences, say $\widetilde{E}_{\infty}^{(n, m)}$, between the analytic and approximate values evaluated at these points.

The numerical parameter for computing the Schottky-Klein prime function is set $N=$ 100 for the domain of Figure 8(a). In the domain of Figure 8(b), we make the circular boundaries close to each other but still away from the coastline. Since the analyticity of the Schottky-Klein prime function is getting worse as the distances between the circular boundaries are close, a large value of $N=400$ is required for computing the SchottkyKlein prime function for the domain of Figure 8(b). The maximum errors $E_{\infty}^{(n, 3)}$ and $\widetilde{E}_{\infty}^{(n, 3)}$ vs. the numbers of the discretization points $n$ for the domain of Figures $8(a, b)$ are shown in Figures 9(a,b), which indicate the spectral convergence of $O\left(e^{-0.11 n}\right)$ and $O\left(e^{-0.054 n}\right)$, respectively. See Figure 9(a,b).

When the circular boundaries approach to the coastline as in Figure 8(c), the computation of the Schottky-Klein prime function no longer converges with $N=400$, but the computation becomes out of memory for $N=800$. On the other hand, since our method still converges, we use the numerical data obtained by using our method with $n=16384$ as the analytic values. The point vortex in the complex potential $W_{D}(z)$ is located at $\alpha_{z}=4$ in this case. The maximum errors in Figure 9(c) still yield the spectral convergence of $O\left(e^{-0.019 n}\right)$ as in Figure 9(c). Thus we conclude that the present method converges spectrally as the number of discretization increases when the all boundaries in the coastal domains are smooth.

Let us discuss why the rate of exponential decay rate given in Figure 9 varies. As shown in $[3,8,17]$, the rate of convergence becomes slow or divergent in many methods when the distances between the boundaries are so close to touch each other. For the domain in Figure 8(a), the discs are well separated from each other, so we get good rate of convergence $O\left(e^{-0.11 n}\right)$ as shown in Figure 9(a). For the domain in Figure 8(b), the discs are close to each other but away from the wall, so the rate of convergence decreases 
to $O\left(e^{-0.054 n}\right)$ as in Figure 9(b). In Figure 8(c), the discs become much close to each other and to the wall, so the rate of convergence is getting worse to be $O\left(e^{-0.019 n}\right)$. See Figure 9(c).

\subsubsection{Convergence and efficiency for domains with many obstacles}

This subsection is concerned with the effect of the number of obstacles on the efficiency and the accuracy of the presented numerical method. We consider a coastal domain with $m$ circular obstacles where the coastline is a straight line. We fix the number of discretizing points on each boundaries $n=2048$ and let the number obstacles $m$ increase. Figures 10(a)-(c) show some examples of coastal domains for $m=93, m=651$ and $m=1953$, respectively. The total CPU time (sec.) required to evaluate the boundary values of the complex potential $W_{D}(z)$ for $\alpha_{z}=1.5+\mathrm{i}$ with respect to the number of obstacles $m$ is shown in 11(a), which clearly shows $O(m \ln m)$ computational costs. We also present the maximum error $E_{\infty}^{(2048, m)}$ and the number of GMRES iterations for various $m$ in Figures 11(b,c), respectively. Let us note that we are unable to compute the complex potential from the analytic formula for large $m$, since the MATLAB code for computing the Schottky-Klein prime function does not converge. Thus we regard the complex potential computed by our method with $n=4096$ as the analytic value. These figures clearly illustrate that both of them almost independent of the number of obstacles $m$ and thus the present numerical method remains efficient regardless of $m$.

\subsubsection{Convergence with respect to smoothness of boundaries}

We consider a coastal domain where the coastline is not $C^{\infty}$. Since we are interested in the part of coastline $L_{0}^{\prime}$ between the points $\pm \mathrm{i}$ as in Figure 3(a), we may assume that $L_{0}^{\prime}$ is sampled by the following 21 points. See Figure 12(a).

$$
L_{0}^{\prime}:\left(0.05 \sin 6 \pi t_{i}, t_{i}\right), \quad t_{i}=1-0.1 \mathrm{i}, \quad i=0,1, \ldots, 20 .
$$

We here propose three methods for interpolating the sample points in $L_{0}^{\prime}$ to observe how the convergence rate of the present method depends on smoothness of boundaries. In the first method, assuming that the coastlines from $0 \pm \mathrm{i} \infty$ to $0 \pm 1 \mathrm{i}$ are straight lines, we interpolate the sample points (38) between $\pm \mathrm{i}$ by the cubic spline as shown in Figure 12(b). Then the boundary condition for the cubic spline is chosen so that the coastline $L_{0}$ becomes $C^{1}$ at the points $\pm \mathrm{i}$. We regard the complex potential computed by our method with $n=16384$ as the analytic value. Figure 13(a) shows the maximum error (37) on the boundary values of the complex potential $W_{D}(z)$ for $\alpha_{z}=1.5+\mathrm{i}$ in a coastal domain with $17 \times 8$ offshore circular boundaries in Figure 12(b) for various $n$, which indicates an algebraic convergence of $O\left(n^{-2}\right)$ as $n \rightarrow \infty$.

In the following two interpolation methods of the coastline $L_{0}$, we first consider the conformal mapping

$$
\xi=\Phi(H(z))=\frac{\mathrm{i} z-\mathrm{i}}{z+1}
$$

that maps the sampling points (38) of Figure 12(a) in the complex $z$-plane to the points close to the lower part of the unit circle in the complex $\xi$-plane as in Figure 12(c). We also pick up additional 19 sampling points along the upper half of the unit circle as we see in Figure 12(d). Thus, we have sampled the 40 points along the closed curve $C_{0}$. Note that the sampling points for the coastline $L_{0}$ are obtained through the inverse of (39).

In the second interpolation method, we use the periodic cubic spline that passes through these 40 points, which gives us a $C^{2}$ curve for $C_{0}$ as well as $L_{0}$. The maximum error vs. the number of discretizing points $n$ in Figure 13(b) indicates an $O\left(n^{-3}\right)$ convergence as $n \rightarrow \infty$. The last method utilizes trigonometric polynomials to an interpolation of the curve $C_{0}$ that passes through the points in Figure 12(d), which gives rise to 
a $C^{\infty}$ curve. The maximum error vs. $n$ in Figure 13(c) then shows a spectral convergence of $O\left(e^{-0.03 n}\right)$ as $n \rightarrow \infty$.

\subsection{Application to real coastal domains}

The present fast numerical conformal mapping methods are applicable to coastal domains with many obstacles of arbitrary shapes. The boundary of the coastline is approximated by using trigonometric interpolating polynomials as explained in $§ 5.3 .3$. Similarly, the boundaries of the obstacles are approximated by using trigonometric interpolating polynomials. It is thus easy to construct the complex potentials in real coastal domains. For example, we consider the flow domain in the vicinity of the Penang Island and the coastline of west Malaysia in Figure 1(a), in which we illustrate streamlines of a uniform flow in Figure 14(a), a flow induced by a source (or a sink) point, which is a model of a river mouth, in Figure 14(b), a flow by a source-sink pair in Figure 14(c), a flow around a point vortex in Figure 14(d) and a flow induced by a vortex dipole in Figure 14(e).

We here observe the behavior of a single point vortex, which is a model of a coherent big ocean eddy, by plotting contour lines of the Robin function (35). We are in particular interested in stagnation points of the point vortex. This is because the motion of a point vortex becomes periodic in the neighborhood of an elliptic stagnation point, while hyperbolic (saddle) stagnation points and their separatrices divide the flow domain into some small subdomains where the qualitative behaviors of a point vortex becomes different. Moreover, we observe how the motion of the point vortex changes in the presence of a uniform flow representing a global ocean current. Specifically, we plot the contour lines of the following function in coastal domains.

$$
H_{K}(\alpha)=-\frac{\kappa^{2}}{2} g(\alpha ; \alpha)+U \operatorname{Im}\left[W_{U}(\alpha)\right]
$$

where $\kappa$ and $U$ denote the strength of the point vortex and the flux of the uniform flow.

Figure 15(a) shows trajectories of a point vortex with $\kappa=1$ near Penang Island and the coastline of west Malaysia in the absence of the uniform flow, i.e. $U=0$. There is a hyperbolic stagnation point between the island and the coastline, whose separatrices give rise to three flow domains. First, there is a narrow band domain along the coastline, in which the point vortex goes along the coastline from bottom to top. Second, there is an annular domain where the point vortex rotates around the island in the clockwise direction. In the third domain, trajectories coming from the bottom along the coastline move around the outer side of the island and then go upward along the coastline. If the uniform flow is included and its flux rate becomes $U=1$, the stagnation point still exists and an elliptic stagnation point gives rise to a small domain of periodic orbits near the upper-left side of the island as shown in Figure 15(b). When we increase the flux up to $U=4$, the domain of periodic orbits disappears and the point vortex is just advected by the uniform flow from bottom to top as we see in Figure 15(c).

Let us finally show another example for trajectories of a point vortex in the flow domain around Japanese islands and the coastline of East Asia in Figure 1(b). Here, we set $\kappa=1$ and just change $U$ in (40). We may assume that the sign of $U$ is non positive, since the uniform flow with $U \leq 0$ goes from south to north, which corresponds to the real global ocean current in this flow domain. Trajectories of the point vortex for $U=0,-0.25,-1$, and -4 are shown in Figure 16(a), (b), (c) and (d), respectively. Without the uniform flow in Figure 16(a), there are many hyperbolic stagnation points between the islands and the coastline, whose separatrices enclose a big domain consisting of periodic orbits in the clockwise direction in the Sea of Japan. The point vortex goes from north to south in the Pacific ocean in the absence of the uniform flow. When the uniform flow with $U=-0.25$ is introduced as in Figure 16(b), the point vortex located far from Japan islands in the Pacific ocean changes its moving direction, while the domain of periodic orbits in the Sea 
of Japan still remains. This indicates that the weak uniform flow prevents the point vortex from getting into the flow domain between Japan island and the coastline. For $U=-1$ in Figure 16(c), periodic orbits are confined in domains near the east side of the coastline and thus most of the point vortices in the Sea of Japan move northward according to the uniform flow from the south. This indicates that the self-induced velocity by the point vortex is unable to overcome the strong uniform flow. When the northward uniform flow is strong enough $U=-4$, the domain of periodic orbits disappears and the point vortex is just advected by the uniform flow as we see in Figure 16(d).

\section{Concluding remarks}

We have developed a fast and accurate numerical method for constructing potential flows and the Kirchhoff-Routh path functions in 2D multiply connected coastal domains with complex topography and high connectivity, in which the numerical conformal mapping method based on the boundary integral equations with the generalized Neumann kernel and its adjoint kernel play a significant role. The numerical conformal mapping method is solved by a fast method in $O((m+1) n \ln n)$ operations where $m+1$ is the multiplicity of the multiply connected coastal domain and $n$ is the number of nodes in the discretization of each boundary component (see also $[23,24]$ ). The method is fast, accurate, flexible, and has the ability to be used for domains with very high connectivity and complex geometry. When the boundaries are $C^{\infty}$, it converges spectrally as $O\left(e^{-c n}\right)$ for a certain constant even if the distances between boundaries are very close. It also shows an algebraic convergence for $C^{q}(q \geq 1)$ boundaries. When the boundaries of the coastline is sampled just by discrete points, we have proposed the three interpolation methods. Using the trigonometric interpolating, we have a spectral convergence of $O\left(e^{-c n}\right)$ where $c$ is a positive real constant. The implementation of the present numerical method is also easy as shown in the following appendices, since we just choose the right-hand side of the integral equation appropriately for each canonical domains. Thus it is possible for us to consider $2 \mathrm{D}$ vortex dynamics in many real geographic flows near a long coastline with many offshore islands, since some of these flows are modelled by coastal domains.

\section{Appendix A. Numerical conformal mapping onto the canonical domains}

Let us review the method presented in $[20,22]$ for numerical construction of the conformal mapping from the multiply connected domain $D$ or $S$ onto the canonical domains $\Omega_{D}$, $\Omega_{S}, \Omega_{U}$ and $\Omega_{B}$ used in Section 4 .

Suppose that each boundary component $\mathcal{C}_{j}$ is parametrized by a continuously differentiable complex function $\xi_{j}(t)$ with $\xi_{j}^{\prime}(t) \neq 0$ for $t \in J_{j}=[0,2 \pi], j=0,1, \ldots, m$ in the complex $\xi$-plane. The whole boundary $\mathcal{C}$ is represented by a smooth function on the disjoint union $J$ of the $m+1$ intervals $J_{0}, J_{1}, \ldots, J_{m}$, which is defined by (see [4, p. 16] and $[14$, p. 394])

$$
J=\bigsqcup_{j=0}^{m} J_{j}=\bigsqcup_{j=0}^{m}\left\{(t, j): t \in J_{j}\right\} .
$$

The elements of $J$ are order pairs $(t, j)$ where $j$ is an auxiliary index indicating which of the intervals the point $t$ lies in. Thus, the parametrization of the whole boundary $\mathcal{C}=\mathcal{C}_{0} \cup \mathcal{C}_{1} \cup \cdots \cup \mathcal{C}_{m}$ is defined on $J$ by

$$
\xi(t, j)=\xi_{j}(t), \quad t \in J_{j}, \quad j=0,1, \ldots, m .
$$

We assume that for a given $t$ that the auxiliary index $j$ is known, so we replace the pair $(t, j)$ in the left-hand side of (42) by $t$, i.e., for a given point $t \in J$, we always know the 
interval $J_{j}$ that contains $t$. The function $\xi$ in (42) is thus simply written as

$$
\xi(t):=\left\{\begin{array}{cc}
\xi_{0}(t), & t \in J_{0}=[0,2 \pi], \\
\xi_{1}(t), & t \in J_{1}=[0,2 \pi], \\
\vdots & \\
\xi_{m}(t), & t \in J_{m}=[0,2 \pi] .
\end{array}\right.
$$

In the construction of the conformal mapping, for a given Hölder continuous real-valued function $\gamma(\xi(t))$ defined on $\mathcal{C}$ and $\alpha \in D$, there appears the following Riemann-Hilbert problem for a function $f(\xi(t))$ and a piecewise constant function $h(\xi(t))$.

$$
\operatorname{Re}[A(\xi(t)) f(\xi(t))]=\gamma(\xi(t))+h(\xi(t)), \quad t \in J,
$$

where $A(\xi)=\xi-\alpha$. Note that the functional form of $\gamma(\xi)$ varies depending on the conformal mappings as shown in Section 4 , and this problem is solvable uniquely according to [19]. When we set $\mu(\xi(t))=\operatorname{Im}[A(\xi(t)) f(\xi(t))]$ for $t \in J$, the boundary value of the function $f$ is given by

$$
A(\xi(t)) f(\xi(t))=\gamma(\xi(t))+h(\xi(t))+\mathrm{i} \mu(\xi(t)), \quad t \in J .
$$

For a Hölder continuous function $\mu$ on $\mathcal{C}$, let us introduce the integral operators $\mathbf{N}$ and $\mathbf{M}$ as

$$
\mathbf{N} \mu=\int_{J} N(s, t) \mu(t) d t, \quad \mathbf{M} \mu=\int_{J} M(s, t) \mu(t) d t,
$$

where the generalized Neumann kernel $N(s, t)$ and the kernel $M(s, t)$ are defined on $J \times$ $J[31]$ as follows.

$$
N(s, t):=\frac{1}{\pi} \operatorname{Im}\left(\frac{A(s)}{A(t)} \frac{\xi^{\prime}(t)}{\xi(t)-\xi(s)}\right), \quad M(s, t):=\frac{1}{\pi} \operatorname{Re}\left(\frac{A(s)}{A(t)} \frac{\xi^{\prime}(t)}{\xi(t)-\xi(s)}\right) .
$$

The operator $\mathbf{N}$ is a Fredholm integral operator and the operator $\mathbf{M}$ is a singular operator, which are bounded on the space of Hölder continuous functions on $\mathcal{C}$ and map this space onto itself [30]. Then the boundary value problem is solved based on the following theorem.

Theorem 1. For any Hölder continuous real-valued function $\gamma$ defined on $\mathcal{C}$, there exists a unique Hölder continuous real-valued function $\mu$ and a unique piecewise constant realvalued function $h=\left(h_{0}, h_{1}, \ldots, h_{m}\right)$ such that $(45)$ are boundary values of analytic function $f$ in $D$. Then function $\mu$ is the unique solution of the integral equation

$$
(\mathbf{I}-\mathbf{N}) \mu=-\mathbf{M} \gamma,
$$

where $\mathbf{I}$ denotes the identity operator and the function $h$ is given by

$$
h=[\mathbf{M} \mu-(\mathbf{I}-\mathbf{N}) \gamma] / 2 .
$$

In practical computations, by discretizing the integral equation (48) by a quadrature rule with $n$ nodes along the $m+1$ boundaries, we have the $(m+1) n$ dimensional linear equations for the boundary values of $\mu$, i.e. the imaginary part of $f$, which is numerically solved fast as explained in Appendix C. We then compute the piecewise constant function $h$ on the boundary by (49). The real part of $f$ is finally computed from (44). 


\section{Appendix B. Numerical conformal mapping onto the domain $S$}

In order to compute the conformal mappings to the canonical domains $\Omega_{U}$ and $\Omega_{B}$, we need to map the domain $D$ to a bounded multiply connected domain $S$ inside the unit circle. This can be done by mapping the simply connected domain inside $C_{0}$ onto the unit disc. Such a mapping function is computed using the method presented in [20, §4.2], which is explained in what follows. The basic idea is the same as the method explained Appendix A. Here, we just care the image of the outer curved boundary $C_{0}$, which is parameterized by $\xi_{0}(t)$ for $t \in J_{0}=[0,2 \pi]$, and we don't need to consider the images of the inner boundaries of $D$.

For a complex-valued function $\AA(t)=\xi_{0}(t)-\alpha_{\xi}$ and the outer curved boundary $\xi_{0}(t)$ for $t \in J_{0}=[0,2 \pi]$ and $\alpha_{\xi} \in D$, let us define the following generalized Neumann kernel $\stackrel{\circ}{N}(s, t)$ and the real kernel $\stackrel{M}{M}(s, t)$ on $J_{0} \times J_{0}[18,30]$.

$$
\stackrel{\circ}{N}(s, t):=\frac{1}{\pi} \operatorname{Im}\left(\frac{\stackrel{\AA}{A}(s)}{\stackrel{\circ}{A}(t)} \frac{\xi_{0}^{\prime}(t)}{\xi_{0}(t)-\xi_{0}(s)}\right), \quad \stackrel{\circ}{M}(s, t):=\frac{1}{\pi} \operatorname{Re}\left(\frac{\stackrel{\AA}{A}(s)}{\stackrel{\AA}{A}(t)} \frac{\xi_{0}^{\prime}(t)}{\xi_{0}(t)-\xi_{0}(s)}\right) .
$$

The Riemann-Hilbert problem (4) in $\S 3.3$ is solved again based on Theorem 1. Namely, for

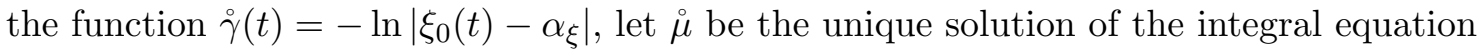

$$
(\mathbf{I}-\stackrel{\circ}{\mathbf{N}}) \stackrel{\circ}{\mu}=-\stackrel{\circ}{\mathbf{M}} \dot{\circ},
$$

in which $\mathbf{I}$ denotes the identity operator and

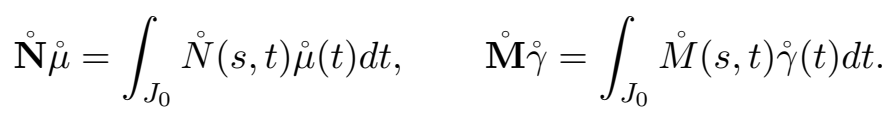

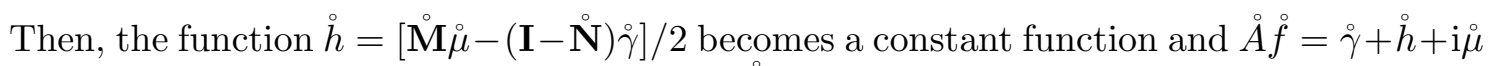
are boundary values of an analytic function $\dot{f}$ on the simply connected domain interior of $C_{0}$.

In the construction of the conformal mapping $\omega_{U}(\eta)$ and $\omega_{B}(\eta)$ with the numerical conformal mapping technique in Appendix A, we need to compute the derivative of the boundaries in the domain $S$, i.e. $\eta_{j}^{\prime}(t)$ for $j=0, \ldots, m$, in order to compute the kernels (47). For the outer boundary $\eta_{0}(t)$, it follows from (5) that it is computed by

$$
\eta_{0}^{\prime}(t)=\Upsilon^{\prime}\left(\xi_{0}(t)\right) \xi_{0}^{\prime}(t)=T^{\prime}\left(R\left(\xi_{0}(t)\right)\right) R^{\prime}\left(\xi_{0}(t)\right) \xi_{0}^{\prime}(t), \quad t \in J_{0} .
$$

The derivative of the conformal mapping $R$ can be computed using a boundary integral equation with the adjoint generalized Neumann kernel [25]. The boundary value of the mapping function $R$ can be written as $[25, \S 6]$

$$
R\left(\xi_{0}(t)\right)=e^{\mathrm{i} \phi(t)}, \quad t \in J_{0},
$$

where $\phi$ is known as the boundary correspondence function. Differentiating both sides of (52) with respect to the parameter $t$, we obtain

$$
\xi_{0}^{\prime}(t) R^{\prime}\left(\xi_{0}(t)\right)=\mathrm{i} \phi^{\prime}(t) e^{\mathrm{i} \phi(t)}, \quad t \in J_{0} .
$$

The function $\phi^{\prime}$ is the unique solution of the integral equation $[25, \S 6]$ :

$$
\left(\mathbf{I}+\stackrel{\circ}{\mathbf{N}}^{*}+\stackrel{\circ}{\mathbf{J}}\right) \phi^{\prime}=1
$$

where $\mathbf{N}^{*}$ is the adjoint operator of the operator $\mathbf{N}$, which is given by

$$
\stackrel{\circ}{\mathbf{N}}^{*} \mu=\int_{J_{0}} \stackrel{\circ}{N}(t, s) \mu(t) d t
$$


and the operator $\mathbf{J}$ is defined by

$$
\stackrel{\circ}{\mathbf{J}} \mu=\frac{1}{2 \pi} \int_{J_{0}} \mu(t) d t .
$$

By solving the integral equation (54), we obtain approximation of the boundary value of $\phi^{\prime}$. Hence, it follows from (51), (52) and (53), that the boundary value of $\Upsilon^{\prime}$ on $C_{0}$ is given by

$$
\Upsilon^{\prime}\left(\xi_{0}(t)\right)=\frac{\mathrm{i} \phi^{\prime}(t) R\left(\xi_{0}(t)\right) T^{\prime}\left(R\left(\xi_{0}(t)\right)\right)}{\xi_{0}^{\prime}(t)}, \quad t \in J_{0},
$$

which yields the derivative $\eta_{0}^{\prime}(t)$ from (51). For $j=1,2, \ldots, m$, the derivatives of the boundaries $\eta_{j}^{\prime}(t)$ are given by $\eta_{j}^{\prime}(t)=\zeta_{j}^{\prime}(t) \Upsilon^{\prime}\left(\zeta_{j}(t)\right)$ for $t \in J_{j}$. Since $\Upsilon$ is analytic in the domain interior to $C_{0}$, in view of (56), the values of $\Upsilon^{\prime}\left(\zeta_{j}(t)\right)$ are computed using Cauchy's integral formula. Thus the boundaries of $\Gamma$ of the domain $S$ is parameterized by $\eta_{j}(t)$, $t \in J_{j}=[0,2 \pi]$ for $j=0,1, \ldots, m$.

\section{Appendix C. Technical notes for solving the integral equation}

The boundary integral equations (48) and (50) can be solved accurately by the Nyström method with the trapezoidal rule (see e.g., $[2,13]$ ). For $j=0,1, \ldots, m$, each interval $J_{j}$ is discretized by $n$ equidistant nodes

$$
s_{j, k}=(k-1) \frac{2 \pi}{n}, \quad k=1,2, \ldots, n .
$$

Hence, the total number of nodes in the total parameter domain $J$ is $(m+1) n$.

The equations (50) and (54) are boundary integral equations on a single boundary component $C_{0}$. The Nyström method with the trapezoidal rule with the $n$ equidistant nodes $s_{0, k}, k=1,2, \ldots, n$, are used to discretize the integral equations (50) and (54) to obtain $n \times n$ linear systems. The equation (48) is a boundary integral equation on $m+1$ boundary components. The Nyström method with the trapezoidal rule with the $(m+1) n$ equidistant nodes $s_{j, k}$ for $j=0,1, \ldots, m$ and $k=1,2, \ldots, n$, are used to discretize the integral equation $(48)$ to obtain $(m+1) n \times(m+1) n$ linear systems (see $[19,20,23,24]$ for details). These linear systems are solved by the generalized minimal residual (GMRES) method [28]. Each iteration of the GMRES method requires a matrix-vector product which can be computed using the Fast Multipole Method (FMM).

The order of the convergence of the Nyström method is based on the order of the convergence of the trapezoidal rule which in turn depends on the smoothness of the integrand. Under suitable regularity assumptions on the integrand, the order of the convergence of the Nyström method is the same as the order of the convergence of the trapezoidal rule (see e.g., [2, p. 109] and [13, p. 227]). If the integrand is $q$ times continuously differentiable, then the rate of convergence of the trapezoidal rule is $O\left(1 / n^{q}\right)$. For $C^{\infty}$ smooth integrands, the rate of convergence is $O\left(e^{-c n}\right)$ for some positive constant $c$ depending on the integrand (see [13, p. 223]). The smoothness of the integrand depends on the smoothness of the boundary of the domain. This convergence property is confirmed clearly in the present paper.

In actual numerical computations, the integral equation (50) is solved using the function FBIE that requires $O(n \ln n)$ operations, while we need $O(n)$ operations to solve the integral equation (54) using the function FBIEad. These functions utilize the MATLAB function gmres and the function zfmm2dpart in the MATLAB toolbox FMMLIB2D developed by Greengard and Gimbutas [10] for the solutions of the linear system and the matrixvector product, respectively. Consequently, the computational cost for solving the integral equation (48) becomes $O((m+1) n \ln n)$, since we use the function FBIE. In the MATLAB functions FBIE and FBIEad, we choose the parameters iprec $=5$, restart $=10$, 
gmrestol $=0.5 \times 10^{-14}$, maxit $=10$, which means that the tolerance of the FMM is $0.5 \times 10^{-15}$, the GMRES method is restarted every 10 inner iterations, the tolerance of the GMRES method is $0.5 \times 10^{-15}$, and the maximum number of outer iterations of GMRES method is 10 . See $[23,24]$ for more details.

\section{References}

[1] K. Amano, A charge simulation method for numerical conformal mapping onto circular and radial slit domains, SIAM J. Sci. Comput., 19 (1998) 1169-1187.

[2] K.E. Atkinson, The Numerical Solution of Integral Equations of the Second Kind, Cambridge University Press, Cambridge, 1997.

[3] A.P. Austin, P. Kravanja and L.N. Trefethen, Numerical algorithms based on analytic function values at roots of unity, SIAM J. Numer. Anal., 52(4) (2014) 1795-1821.

[4] F. Bullo and A.D. Lewis, Geometric control of mechanical systems, Springer, New York, 2005.

[5] D. Crowdy and J. Marshall, Analytic formulae for the Kirchhoff-Routh path function in multiply connected domains, Proc. Roy. Soc. A, 461 (2005) 2477-2501.

[6] D. Crowdy and J. Marshall, The motion of a point vortex around multiple circular islands, Phys. Fluids, 17 (2005) 056602.

[7] D. Crowdy and J. Marshall, Computing the Schottky-Klein prime function on the Schottky double of planar domains, Comput. Methods Funct. Theory, 7(1), 293308, (2007). MATLAB files available in: http://www2.imperial . ac .uk/ dgcrowdy/ SKPrime.

[8] D.G. Crowdy, S. Tanveer and T. Delillo, Hybrid Basis Scheme for computing Electrostatic fields exterior to close-to-touching discs, arXiv preprint arXiv:1409.2466 (2014).

[9] D. Fratantoni, W.E. Johns and T. Townsend, Rings of the north Brazil current: their structure and behavior inferred from observations and a numerical simulations, $J$. Geophys. Res., 100 (1995) 10,633-10,654.

[10] L. Greengard and Z. Gimbutas, FMMLIB2D: A MATLAB toolbox for fast multipole method in two dimensions, Version 1.2, 2012. http://www.cims.nyu.edu/cmcl/ fmm2dlib/fmm2dlib.html.

[11] E.R. Johnson and N.R. McDonald, The motion of a vortex near a gap in a wall, Phys. Fluids, 16 (2004) 462-469. doi:10.1063/1.1637603

[12] E.R. Johnson and N.R. McDonald, The motion of a vortex near two circular cylinders, Proc. Roy. Soc. A, 460 (2004) 939-954. doi: 10.1098/rspa.2003.1193

[13] R. Kress, Linear Integral Equations, $3^{\text {rd }}$ ed., Springer, New York, 2014.

[14] J.M. Lee, Introduction to Topological Manifolds, Springer, New York, 2000.

[15] C.C. Lin, On the motion of vortices in two dimensions. I. Existence of the KirchhoffRouth function, Proc. Natl. Acad. Sci., 27 (1941) 570-575.

[16] C.C. Lin, On the motion of vortices in two dimensions. II. Some further investigations on the Kirchhoff-Routh function, Proc. Natl. Acad. Sci., 27 (1941) 575-577. 
[17] V. Mityushev and N. Rylko, A fast algorithm for computing the flux around nonoverlapping disks on the plane, Mathematical and Computer Modelling, 57(5) (2013) $1350-1359$.

[18] A.H.M. Murid and M.M.S. Nasser, Eigenproblem of the generalized Neumann kernel, Bull. Malays. Math. Sci. Soc. (2), 26 (2003) 13-33.

[19] M.M.S. Nasser, A boundary integral equation for conformal mapping of bounded multiply connected regions, Comput. Methods Funct. Theory, 9 (2009) 127-143.

[20] M.M.S. Nasser, Numerical conformal mapping via a boundary integral equation with the generalized Neumann kernel, SIAM J. Sci. Comput., 31(3) (2009) 1695-1715.

[21] M.M.S. Nasser, Numerical conformal mapping of multiply connected regions onto the second, third and fourth categories of Koebe's canonical slit domains, J. Math. Anal. Appl., 382 (2011) 47-56.

[22] M.M.S. Nasser, Numerical conformal mapping of multiply connected regions onto the fifth category of Koebe's canonical slit regions, J. Math. Anal. Appl., 398 (2013) $729-743$.

[23] M.M.S. Nasser and F.A.A. Al-Shihri, A fast boundary integral equation method for conformal mapping of multiply connected regions, SIAM J. Sci. Comput., 35(3) (2013) A1736-A1760.

[24] M.M.S. Nasser, Fast solution of boundary integral equations with the generalized Neumann kernel, arXiv:1308.5351, 2013.

[25] M.M.S. Nasser, A.H.M. Murid, and A.W.K. Sangawi, Numerical conformal mapping via a boundary integral equation with the adjoint generalized Neumann kernel, TWMS J. Pure Appl. Math., 5(1) (2014) 96-117.

[26] P.L. Richardson and A. Tychensky, Meddy trajectories in the Canary Basin measured during the semaphore experiment, 1993-1995, J. Geophys. Res., 103 (1998) 25,02925,045 .

[27] E.J. Routh, Some applications of conjugate functions, Proc. Lond. Math. Soc., 12 (1881) 73-89.

[28] Y. Saad and M.H. Schultz, GMRES: A generalized minimum residual algorithm for solving nonsymmetric linear systems, SIAM J. Sci. Stat. Comput., 7(3) (1986) 856-869.

[29] T. Sakajo and Y. Amaya, Numerical construction of potential flows in multiply connected channel domains, Comput. Methods Funct. Theory, 11(2) (2011) 415-438.

[30] R. Wegmann, A.H.M Murid, and M.M.S. Nasser, The Riemann-Hilbert problem and the generalized Neumann kernel, J. Comput. Appl. Math., 182 (2005) 388-415.

[31] R. Wegmann and M.M.S. Nasser, The Riemann-Hilbert problem and the generalized Neumann kernel on multiply connected regions, J. Comput. Appl. Math., 214 (2008) $36-57$.

[32] A.A.M. Yunus, A.H.M. Murid, and M.M.S. Nasser, Numerical conformal mapping and its inverse of unbounded multiply connected regions onto logarithmic spiral slit regions and rectilinear slit regions, Proc. Roy. Soc. A., 470.2162 (2014) 20130514. 
(a)

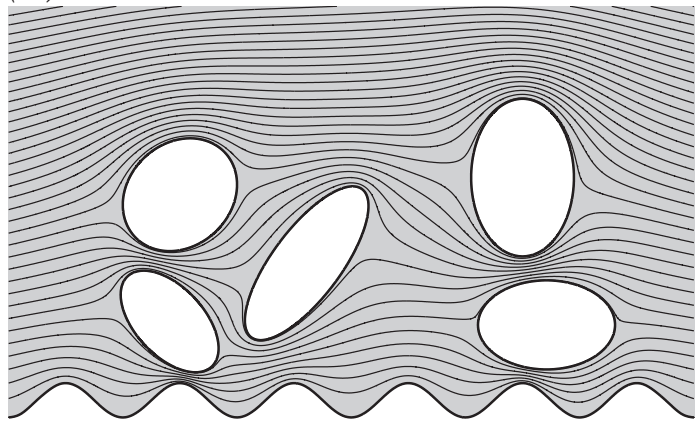

(b)

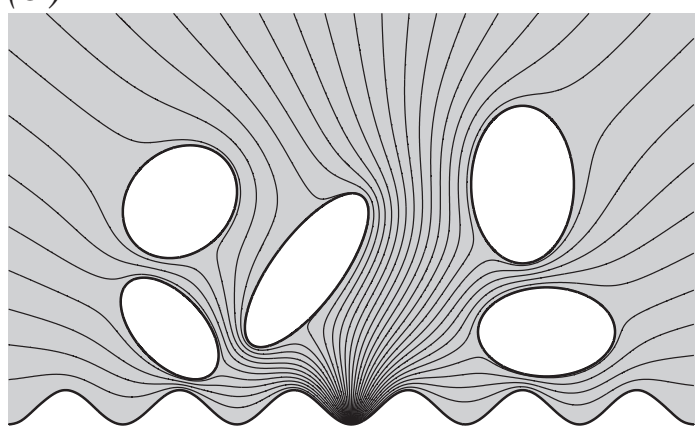

(c)

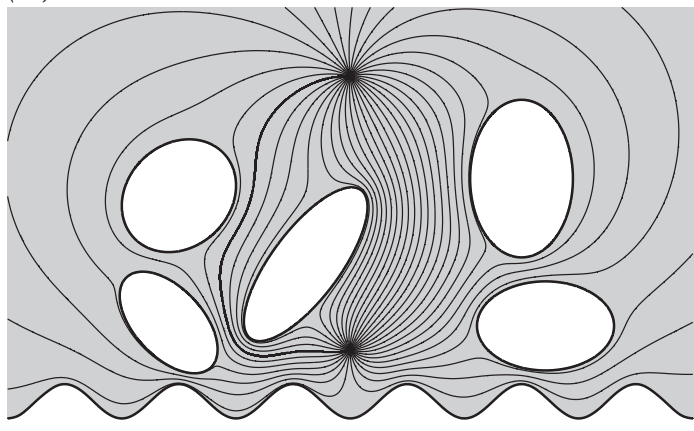

(d)

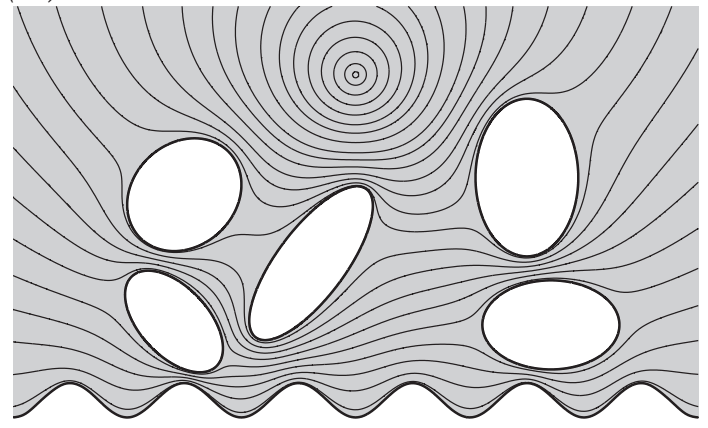

(e)

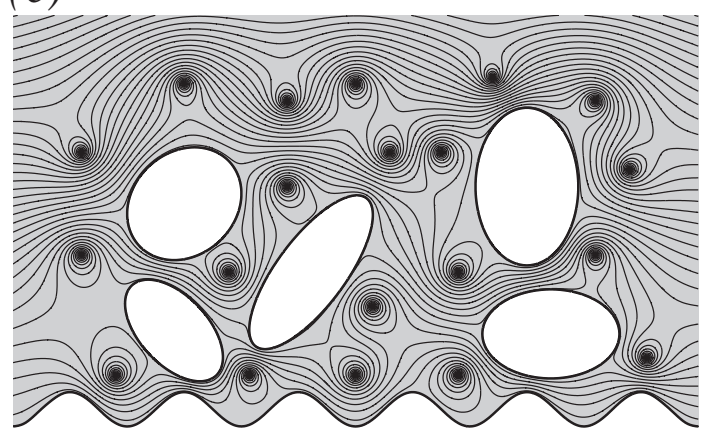

$(f)$

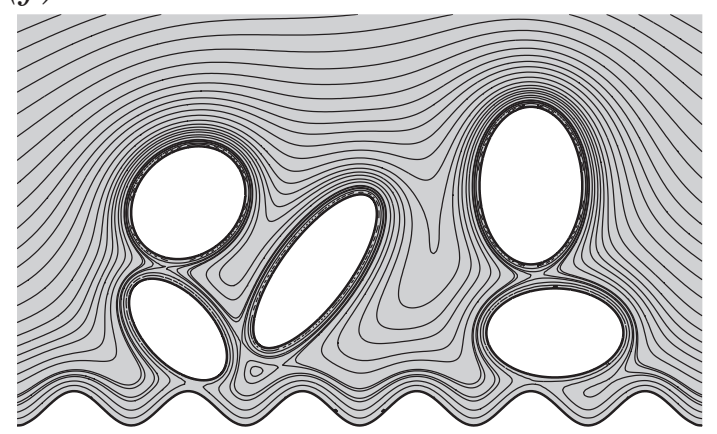

Figure 7: Potential flows in a coastal domain with five elliptic obstacles. (a) Streamlines of a uniform flow. (b) Streamlines of a source point on the wall boundary. (c) Streamlines of a source-sink pair. (d) Streamlines of a point vortex. (e) Streamlines of a uniform flow with 20 point vortices whose complex potential is given by (28). (f) Trajectories of a point vortex. 

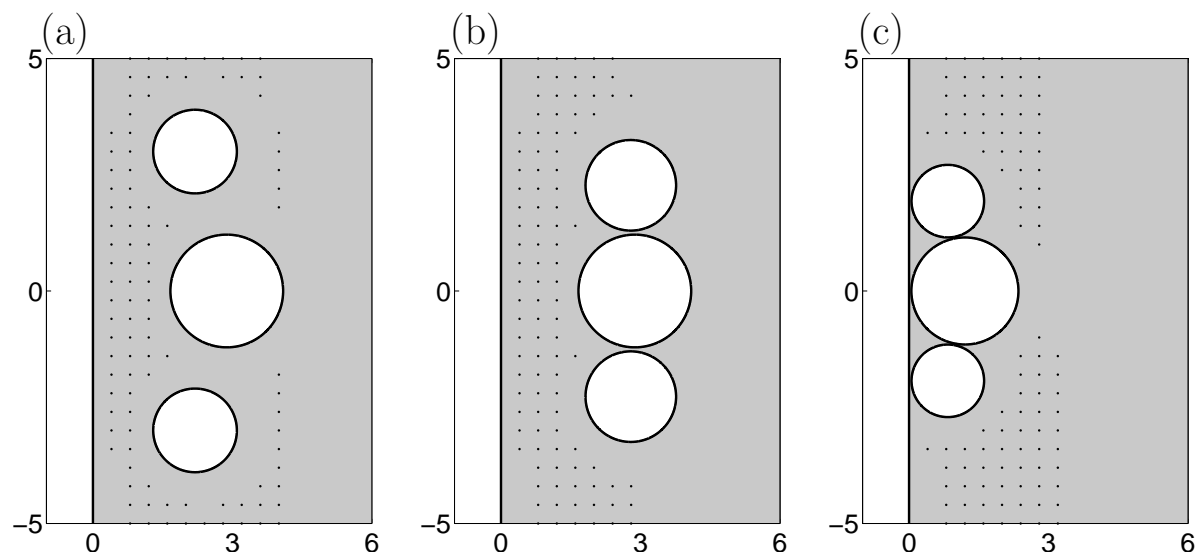

Figure 8: The pre-images under the Möbius transformation (36) of the circular regions interior to the unit circle with three inner circular boundaries, whose centers $q_{i} \in \mathbb{C}$ and radii $r_{i} \in \mathbb{R}$ for $i=1,2,3$ are given by (a) $q_{1}=0.5-0.2 \mathrm{i}, q_{2}=-0.5-0.2 \mathrm{i}, q_{3}=-0.3 \mathrm{i}$, and $r_{1}=r_{2}=0.15, r_{3}=0.2$; (b) $q_{1}=0.35-0.2 \mathrm{i}, q_{2}=-0.35-0.2 \mathrm{i}, q_{3}=-0.3 \mathrm{i}$ and $r_{1}=r_{2}=0.15, r_{3}=0.2$; (c) $q_{1}=0.52-0.57 \mathrm{i}, q_{2}=-0.52-0.57 \mathrm{i}, q_{3}=-0.67 \mathrm{i}$ and $r_{1}=r_{2}=0.21, r_{3}=0.31$, respectively. Dots represent the points where we evaluate the Kirchhoff-Routh path function.
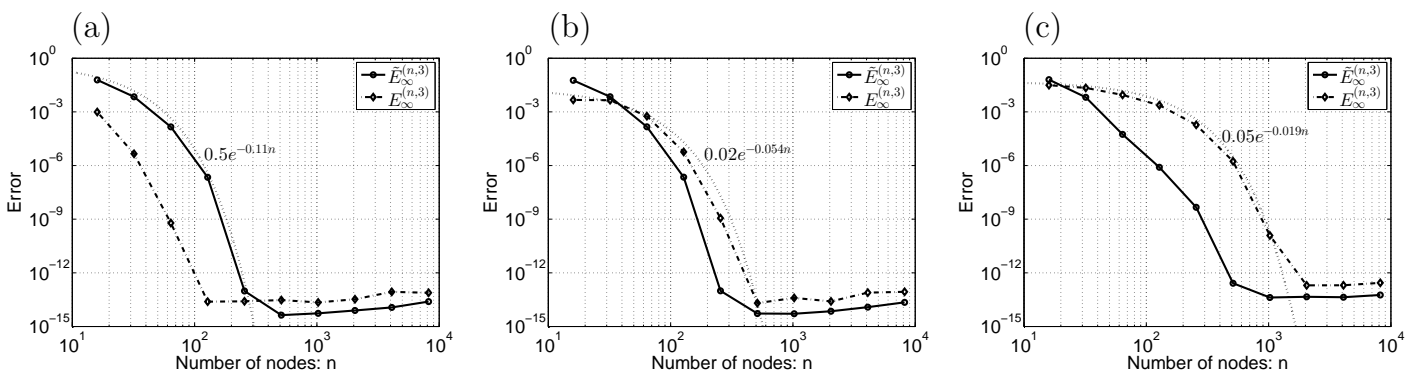

Figure 9: The maximum errors vs. the discretization points $n$ for the multiply connected coastal domains shown in Figure 8.

(a)

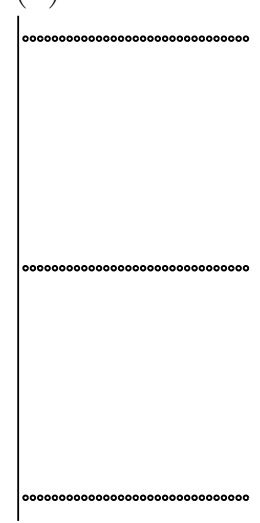

(b)

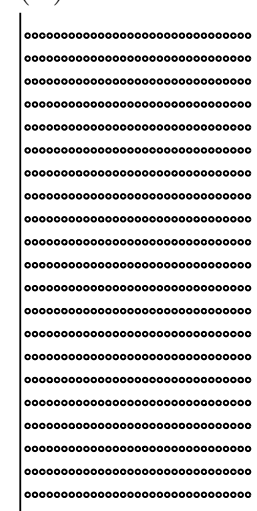

(c)

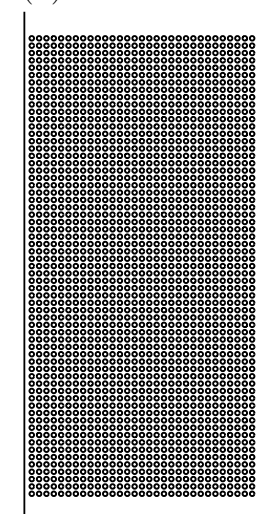

Figure 10: Multiply connected coastal domains with a straight coastline and $m$ offshore circular islands; (a) $m=93$, (b) $m=651$ and (c) $m=1953$. 
(a)

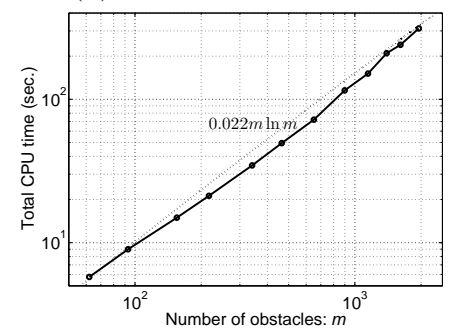

(b)

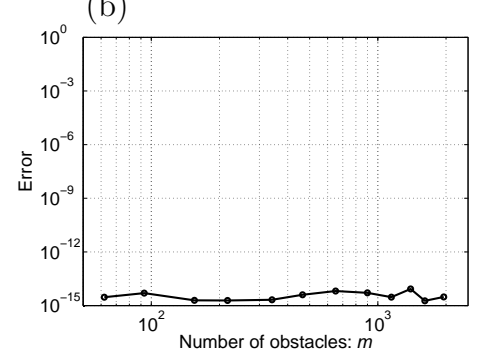

$(\mathrm{c})$

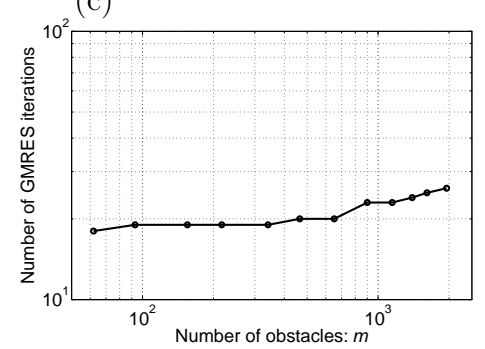

Figure 11: Efficiency of the present numerical algorithm with respect to the number of circular boundaries $m$ in the coastal domains in Figure 10. (a) Total computational time; (b) The maximum error $E_{\infty}^{(2048, m)}$; (c) The number of iterations for GMRES solver.

(a)

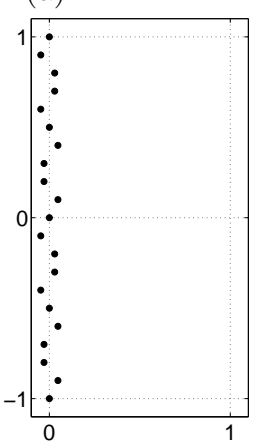

(b)

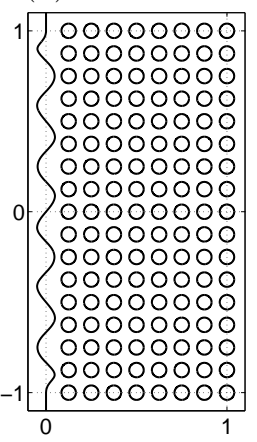

(c)

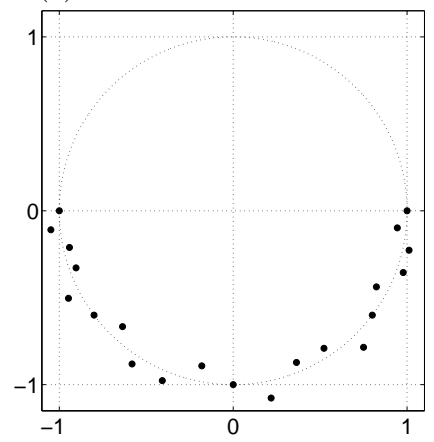

(d)

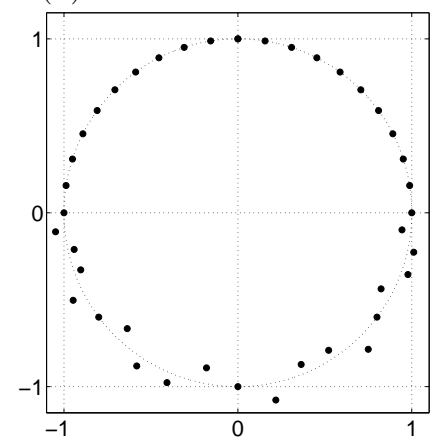

Figure 12: The interpolations of the coastline $L_{0}$. (a) Discrete sampling points along the coastline $L_{0}^{\prime}$ between the points $\pm \mathrm{i}$ in the complex $z$-plane. (b) A coastal domain with a coastline interpolated by the cubic spline and $17 \times 8$ offshore circular boundaries. The images of the sampling points owing to the conformal mapping (39) (c) for the lower half circle and (d) for the full circle in the complex $\xi$-plane.

(a)

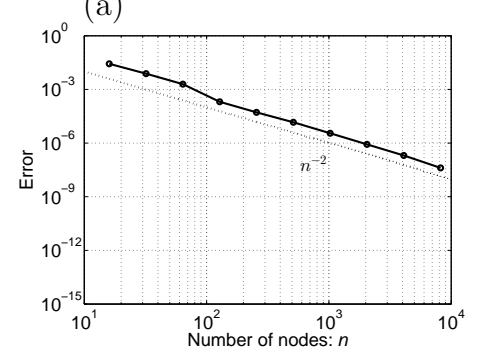

(b)

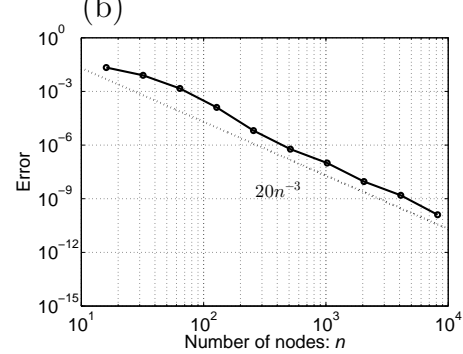

(c)

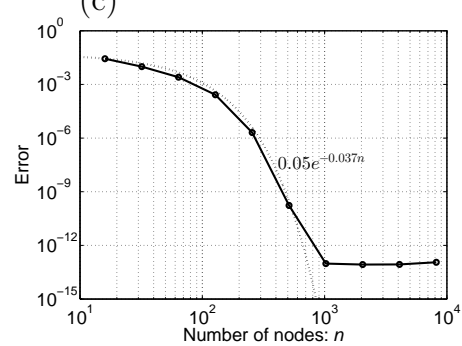

Figure 13: Convergence of the maximum error (37) for the complex potential $W_{D}(\zeta)$ for $\alpha_{z}=1.5+\mathrm{i}$ in the coastal domain with a coastline approximated by discrete points in Figure 12(a). The coastline is interpolated by (a) the cubic spline, (b) the periodic cubic spline, and (c) a trigonometric polynomial. 
(a)

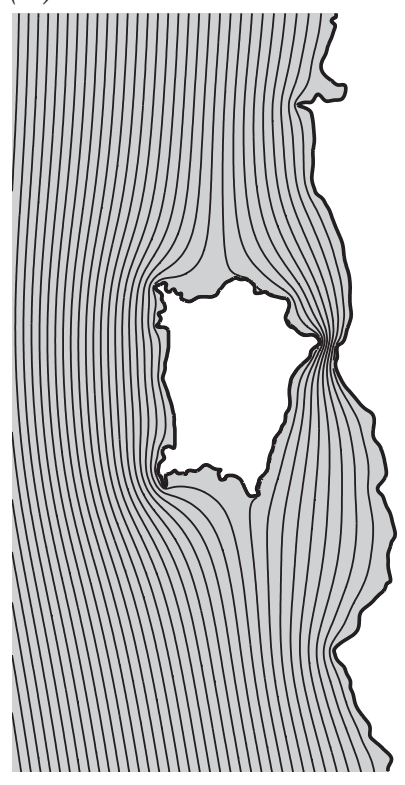

(b)

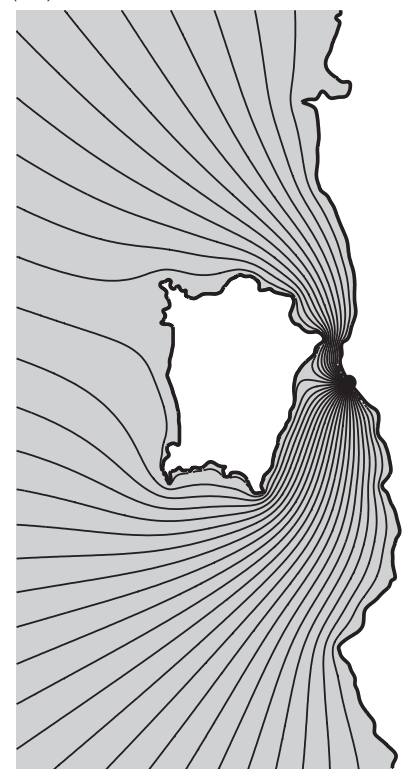

(c)

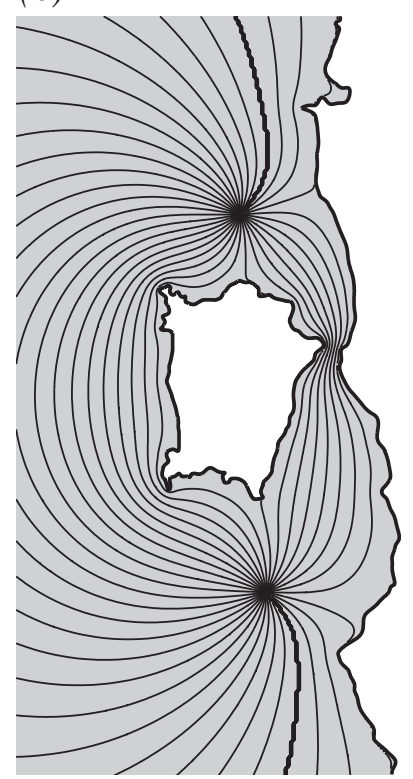

(d)

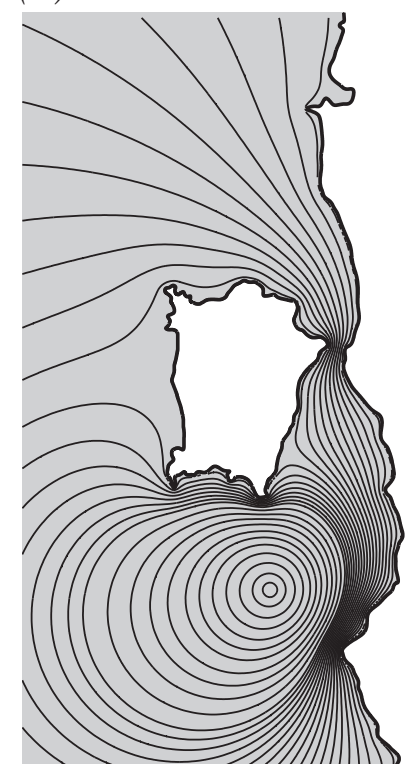

(e)

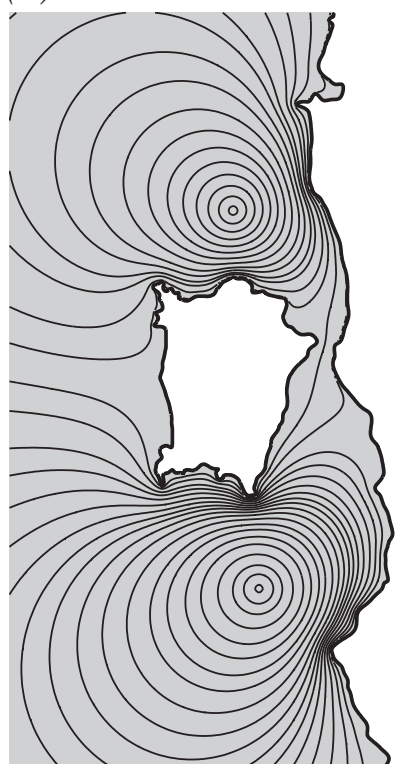

Figure 14: Streamlines in the vicinity of Penang Island and the coastline of west Malaysia. (a) A uniform flow. (b) A flow induced by a source (or a sink) on the coastline. (c) A flow of a source-sink pair. (d) A flow around a point vortex. (e) A flow induced by a vortex dipole. 
(a)

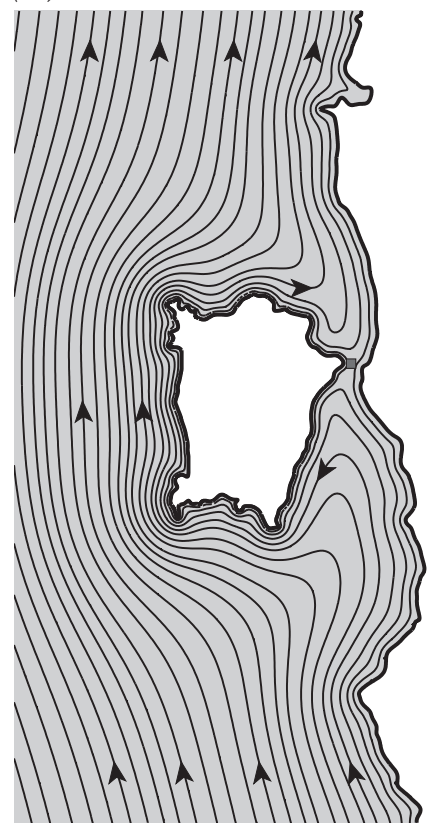

(b)

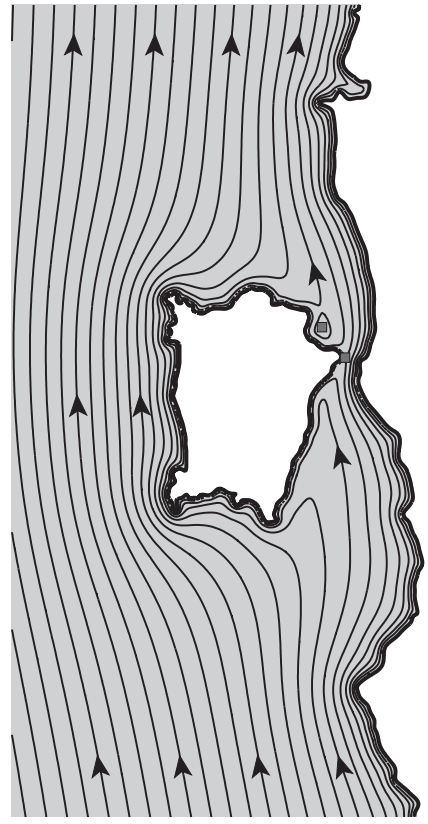

(c)

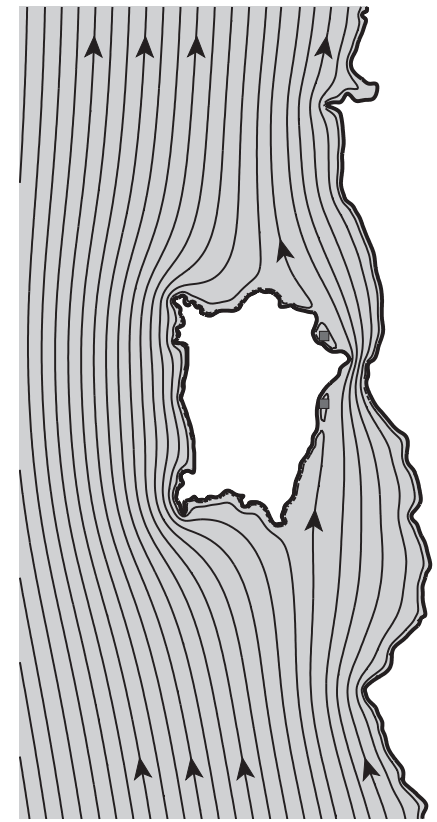

Figure 15: Trajectories of a point vortex in the vicinity of Penang Island and the coastline of west Malaysia. Stagnation points are drawn as gray rectangles. 

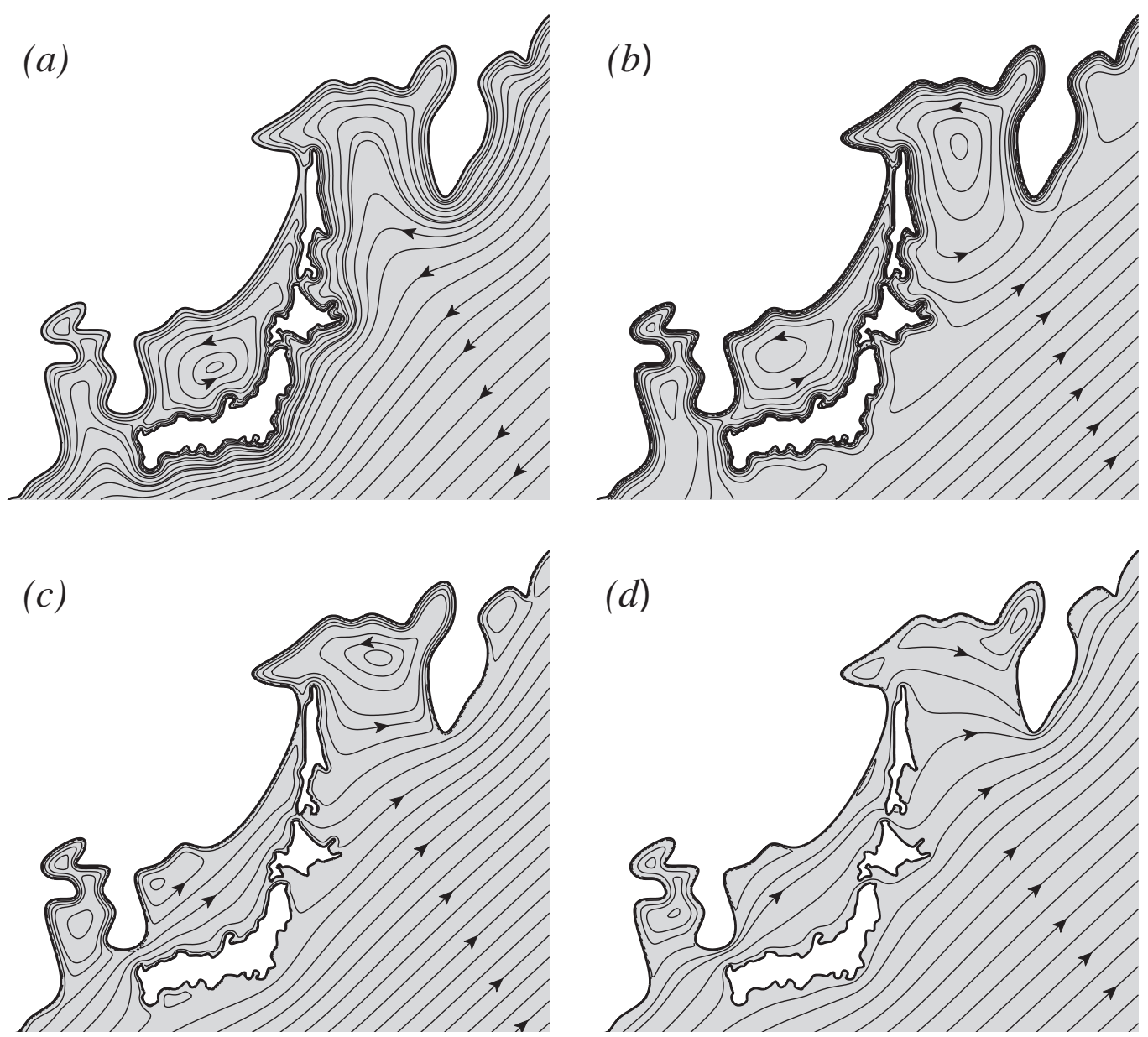

Figure 16: Trajectories of a point vortex with the strength $\kappa=1$ in the vicinity of many islands and the coastline of East Asia in the presence of the uniform flow of (a) $U=0$, (b) $U=-0.25$, (c) $U=-1$, (d) $U=-4$. 